DOCUMENTOS OCASIONALES

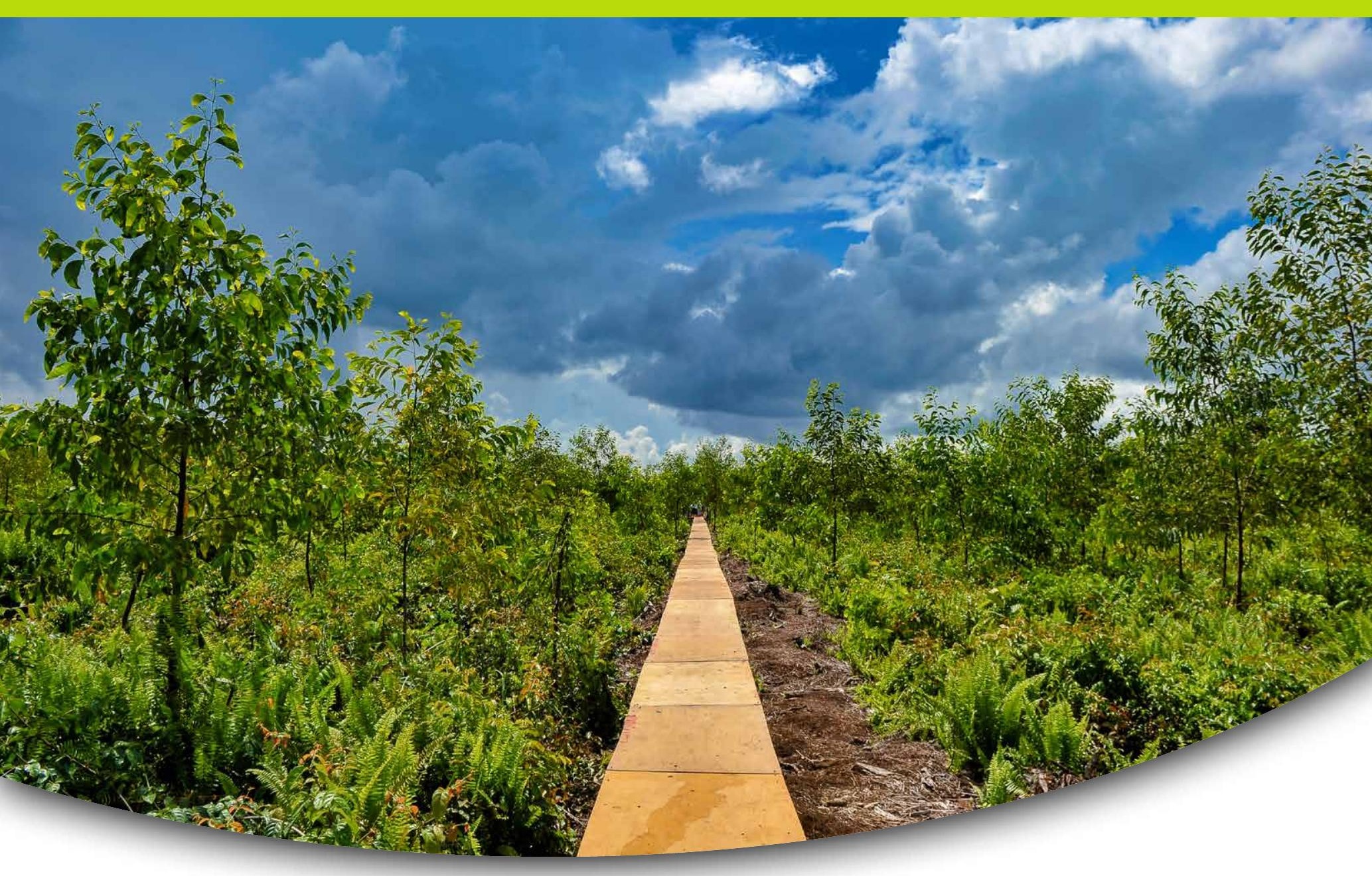

\title{
Un diagnóstico para el monitoreo colaborativo en la restauración de paisajes forestales
}

Kristen Evans

Manuel R Guariguata
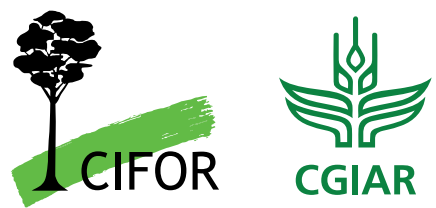



\section{Un diagnóstico para el monitoreo colaborativo en la restauración de paisajes forestales}

Kristen Evans

CIFOR

Manuel R Guariguata

CIFOR 
Documentos Ocasionales 195

(C) 2019 Centro para la Investigación Forestal Internacional (CIFOR)

(c) (i) Los contenidos de esta publicación están bajo licencia Creative Commons Attribution 4.0 International (CC BY 4.0), http://creativecommons.org/licenses/by/4.0/

ISBN 978-602-387-118-6

DOI: $10.17528 /$ cifor/007276

Evans K y Guariguata MR. 2019. Un diagnóstico para el monitoreo colaborativo en la restauración de paisajes forestales. Documentos Ocasionales 195. Bogor, Indonesia: CIFOR.

Traducción de: Evans K and Guariguata MR. 2019. A diagnostic for collaborative monitoring in forest landscape restoration. Occasional Paper 193. Bogor, Indonesia: CIFOR.

Foto de portada por Ricky Martin/CIFOR

Restauración de turberas, Indonesia.

CIFOR

Jl. CIFOR, Situ Gede Bogor Barat 16115 Indonesia

$\mathrm{T}+62(251) 8622-622$

$F+62(251) 8622-100$

E cifor@cgiar.org

\section{cifor.org}

Quisiéramos agradecer a todos los socios financieros que apoyaron esta investigación a través de sus contribuciones al Fondo de CGIAR. Para ver la lista de donantes del Fondo, visite: http://www.cgiar.org/our-funders/

Cualquier opinión vertida en este documento es de los autores. No refleja necesariamente las opiniones de CIFOR, de las instituciones para las que los autores trabajan o de los financiadores. 


\section{Contenido}

Agradecimientos iv

Resumen ejecutivo $\quad \mathbf{v}$

1 Introducción $\quad 1$

1.1 Restauración del paisaje forestal (RPF) 1

1.2 La razón de este diagnóstico 1

2 El diagnóstico $\quad 3$

2.1 La escala temporal 4

2.2 La escala de gobernanza 4

2.3 Motivar la participación local 4

2.4 Asignando los puntajes 4

3 Matriz de factores de éxito esenciales $\quad 6$

4 Glosario de conceptos y definiciones $\quad 11$

$\begin{array}{ll}\text { Referencias } & 13\end{array}$

Apéndice

1 Métodos 16

2 Enfoques de puntuación y recursos de evaluación 18

3 Lista ampliada de factores de éxito 19

\section{Lista de figura, tabla y recuadro}

\section{Figura}

1 Hoja de ruta hacia factores de éxito para el monitoreo colaborativo en la RPF 3

Tabla

1 Matriz de factores de éxito esenciales $\quad 7$

Recuadro

1 Los seis principios de la RPF (tomado de Besseau et al. 2018) 


\section{Agradecimientos}

Con gratitud reconocemos el aporte de Pedro Brancalion, James Reed y Rafael Chaves a la revisión del concepto inicial de este documento. También agradecemos a las siguientes personas por sus contribuciones durante la revisión para priorizar y comentar el conjunto inicial de factores de éxito: Rafael Chaves, Pedro Brancalion, James Reed, Ricardo Viani, Sabin Ray, Wilson Ramirez, Manuel
Peralvo, Cesar Sabogal, Rhett Harrison, Mercy Derkyi, Carol J Pierce Colfer, John Stanturf, Karen Holl, John Parrotta, Liz Ota, Julia Quaedvlieg, Rebecca McLain, Wil de Jong, Manuel Boissière, Daniella Schweizer y Luciana Porter-Bolland. A todos ellos, nuestro agradecimiento sincero por su tiempo y dedicación. Agradecemos igualmente a Sarah Wilson por la revisión del primer borrador. 


\section{Resumen ejecutivo}

Para planificar una iniciativa de restauración del paisaje forestal (RPF) de manera colaborativa y llevarla a cabo con éxito, se requiere de un compromiso a largo plazo por parte de una variedad de partes interesadas. Esto solo es posible cuando las personas involucradas, ya sea que se trate de propietarios de tierras, grupos indígenas, entidades gubernamentales, organizaciones no gubernamentales u otros actores esenciales, se unen para definir objetivos comunes y monitorear el progreso hacia los objetivos trazados. El monitoreo colaborativo puede desempeñar un papel crucial en estos procesos al proporcionar una manera estructurada de incluir a diversos interesados en la RPF, generar la participación local y catalizar el aprendizaje social. Sin embargo, el monitoreo colaborativo es un aspecto nuevo para muchas personas a cargo de la planeación de la RPF, que, si bien podrían estar interesadas en implementar el monitoreo colaborativo, podrían desconocer por dónde empezar.

Este diagnóstico brinda una forma sistemática con la que los planificadores de la RPF pueden evaluar sus iniciativas mediante una lista de factores de éxito. El diagnóstico ayuda a los profesionales a: (1) determinar si están listos para iniciar un monitoreo colaborativo; (2) identificar los elementos que necesitan fortalecer; y (3) evaluar si los sistemas de monitoreo colaborativo existentes van bien encaminados. El diagnóstico puede aplicarse por lo menos en dos escalas: incluye factores a emplear en un sitio específico de RPF, y describe los factores intrínsecos en un sistema de monitoreo colaborativo de múltiples niveles. Consiste de una matriz principal de 42 factores de éxito, además de sugerencias para realizar la evaluación. 



\section{Introducción}

Tanto el compromiso como el apoyo político e institucional a la restauración del paisaje forestal (RPF) han ganado impulso, y quienes toman las decisiones en la RPF a nivel global y local reconocen de manera creciente el papel central del monitoreo colaborativo (denominado también monitoreo participativo ${ }^{1}$ ) para asegurar el éxito de la restauración (Edwards et al. 2017; Mansourian et al. 2017; USDA Forest Service 2017). El monitoreo colaborativo se refiere a un compromiso continuo entre investigadores y pobladores locales en la recopilación y el uso de información para la toma de decisiones a múltiples niveles (Cash et al. 2006; Danielsen et al. 2009; Newig et al. 2010). El monitoreo colaborativo juega un papel crucial al brindar responsabilidad, generar participación local y catalizar el aprendizaje en sistemas de monitoreo. Además, los pobladores locales pueden recolectar información precisa de los cambios en el bosque, de las causas de dichos cambios, de las amenazas a la reforestación, y de los impactos biofísicos y socioeconómicos que la teledetección con frecuencia no registra, lo cual a su vez, y luego de entrenamiento adecuado y de seguimiento, se puede lograr con una tercera parte del costo de los honorarios profesionales. Sin embargo, el monitoreo colaborativo no es una panacea: requiere de inversión y personal para desarrollar capacidades e infraestructura de información, y para proporcionar capacitación y apoyo de seguimiento que garanticen que el monitoreo se realice correctamente y que luego se comparta con frecuencia en múltiples niveles para generar aprendizaje social y una toma de decisiones en colaboración.

1 En lugar del término empleado en publicaciones anteriores, en este documento usamos el término colaborativo en lugar de participativo. Para una explicación sobre este cambio, véase la sección Conceptos y definiciones.

\subsection{Restauración del paisaje forestal (RPF)}

La RPF es un "proceso planificado orientado a recuperar la integridad ecológica y mejorar el bienestar humano en paisajes deforestados o degradados" (Mansourian et al. 2005), el cual reconoce que habrá ventajas y desventajas que se compensen en un paisaje con el fin de alcanzar una diversidad de objetivos. La RPF no busca reforestar el paisaje en su totalidad, ni tampoco implementar solamente enfoques de monoproducción. Más bien, la RPF integra una variedad de intervenciones (agrobosques, bosques de galería, y bosques de producción y de protección, incluyendo las áreas de bosque protegido) en un paisaje más amplio. La implementación de las acciones de RPF se guía por un conjunto de seis principios (véase el recuadro 1 ).

\subsection{La razón de este diagnóstico}

Quienes planean y quienes implementan la RPF y buscan adoptar un monitoreo colaborativo, ya sea solo en un lugar o a lo largo de varios sitios, necesitan saber qué elementos o condiciones deben existir para afianzar el monitoreo colaborativo. De igual forma, de no existir los elementos cruciales, los planificadores necesitan entender qué deben hacer para prepararse. Este diagnóstico fue desarrollado para ayudar a estas personas en dicho proceso mediante la identificación sistemática de factores que (1) existen, y/o (2) deben fortalecerse para implementar el monitoreo colaborativo en la RPF. No se trata de obtener el mejor puntaje, sino de usar este diagnóstico como un mecanismo para mejorar. El diagnóstico puede ser empleado solo como una herramienta de monitoreo para calificar el progreso hacia el logro de objetivos cruciales en varias etapas del monitoreo colaborativo. En otras palabras, puede servir para ayudar a 
Recuadro 1. Los seis principios de la RPF (tomado de Besseau et al. 2018).

1. Enfocarse en los paisajes

2. Involucrar a actores interesados y apoyar la gobernanza participativa

La RPF se realiza al interior y a través de la totalidad de los paisajes, no en sitios individuales, representando mosaicos de usos de tierra que interactúan y prácticas de manejo bajo varios sistemas de tenencia y de gobernanza. Es a esta escala que se pueden balancear las prioridades de tipo ecológico, social y económico

La RPF involucra activamente a actores interesados de diferentes escalas, incluyendo los grupos vulnerables, en la planeación y toma de decisiones concernientes al uso de la tierra, objetivos y estrategias de restauración, métodos de implementación, repartición de beneficios, y procesos de monitoreo y revisión

3. Restaurar múltiples funciones para beneficios múltiples
Las intervenciones de RPF están orientadas a restaurar múltiples funciones ecológicas, sociales y económicas a lo largo de un paisaje y generar una serie de bienes del ecosistema que beneficien a múltiples grupos de actores interesados
4. Mantener y mejorar los ecosistemas naturales en los paisajes

5. Adaptarse al contexto local mediante una variedad de enfoques
La RPF no conlleva a la conversión o destrucción de bosques naturales o de otros ecosistemas. Mejora la conservación, recuperación y manejo sostenible de bosques y otros ecosistemas

La RPF emplea una variedad de enfoques que se adaptan a nivel local a los valores y necesidades de tipo social, cultural, económico y ecológico, y a la historia del paisaje. Se basa en el conocimiento científico más reciente y en las mejores prácticas, y en los conocimientos tradicionales e indígenas, y aplica esa información en el contexto de las capacidades locales y de las estructuras de gobierno existentes o nuevas

La RPF busca mejorar la resiliencia del paisaje y de los actores interesados a mediano y largo plazo. Los enfoques de restauración deben mejorar la diversidad de especies y genética, y deben ajustarse a lo largo del tiempo de forma que reflejen tanto los cambios en el clima como otras condiciones ambientales, de conocimiento, capacidades, necesidades de los actores interesados y de valores sociales. En la medida en que la restauración progrese, deben integrarse la información de las actividades de monitoreo, de la investigación y las recomendaciones de los actores interesados en los planes de manejo "monitorear al monitor" con el fin de verificar que el monitoreo colaborativo esté siendo implementado correctamente. El usuario al que se orienta este diagnóstico es un profesional o un equipo interdisciplinario con experiencia en métodos participativos, restauración forestal y en el monitoreo del manejo de recursos naturales.
El diagnóstico puede aplicarse al menos en dos escalas al incluir factores a emplearse en un lugar específico de RPF y al describir los factores que son intrínsecos en un sistema de monitoreo colaborativo con múltiples niveles. Los factores seleccionados a ser evaluados dependen de los objetivos del usuario. 


\section{El diagnóstico}

Los factores de éxito se obtuvieron a partir de una revisión de más de 80 publicaciones sobre monitoreo participativo y colaborativo (véase el apéndice 1). Los resultados se agruparon y sintetizaron en una serie de aseveraciones que podrían incluirse en cuestionarios, entrevistas o talleres. Luego, los factores de éxito se sometieron a la evaluación de 20 expertos internacionales en el tema y se ordenaron de acuerdo a su utilidad, relevancia e importancia. De manera seguida, los factores de éxito se sintetizaron en una matriz, organizada en escalas temporales y de gobernanza (véase la figura 1 para una hoja de ruta de la matriz). En la tabla 1 se presentan los primeros 42 factores de éxito, los cuales se consideran esenciales. La lista inicial completa se encuentra en el apéndice 3.

Para fines prácticos, la matriz organiza los factores de éxito en un formato de lista. Sin embargo, también es importante saber que hay vínculos e interacciones cruciales entre estos, así como prioridades, situaciones de dependencia y cuellos de botella. Por ejemplo, que la población local quiera participar o no, podría depender de varios aspectos relacionados con el acceso a los recursos, la tenencia, las actitudes de funcionarios locales, el entrenamiento, la gobernanza u otros temas que no se incluyen en la matriz. El usuario debe tener en cuenta que estas realidades complejas algunas veces son la razón subyacente de un factor de éxito $y$, cuando sea posible, identificar y observar estas complejidades podría ayudar a que la RPF en un sito mejore o a que se aborden estos temas.

Es vital identificar la escala en la que operan los factores de éxito para así establecer cuándo y dónde son relevantes, y cómo evaluarlos. Las siguientes dos secciones abordan estos conceptos en detalle.

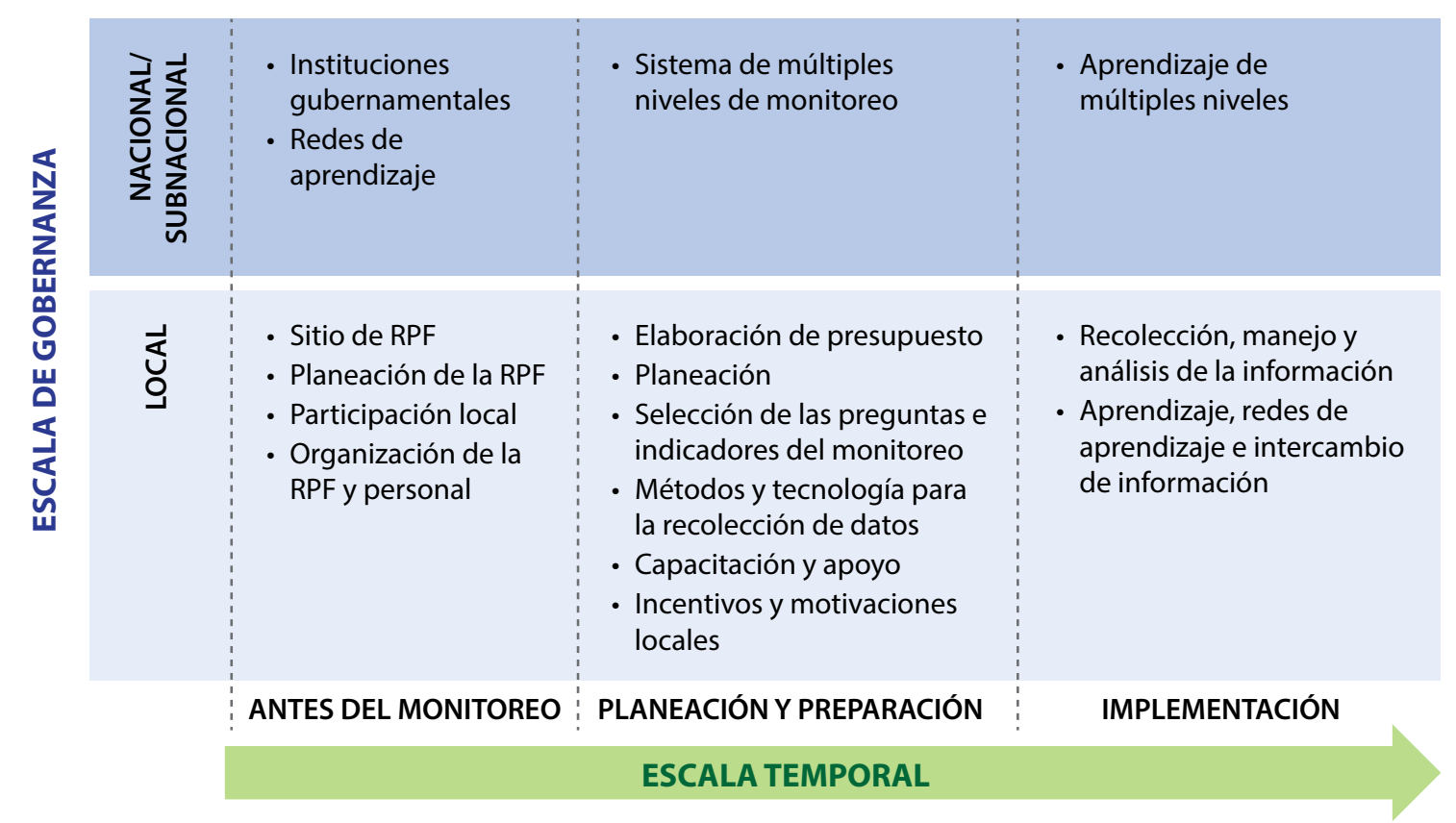

Figura 1. Hoja de ruta hacia factores de éxito para el monitoreo colaborativo en la RPF. Los factores de éxito en la matriz (véase la tabla 1) están organizados en las viñetas. 


\subsection{La escala temporal}

El objetivo de establecer una escala temporal es captar los factores de éxito relevantes para el monitoreo colaborativo a lo largo del ciclo de vida de una iniciativa de RPF, comenzando con una fase de "monitoreo previo", seguida de "planificación" y luego de "implementación". Estas fases posteriores incluyen factores de éxito de un sistema de monitoreo colaborativo ya implementado, no solo las condiciones previas existentes. Esto no quiere decir que al comienzo de una iniciativa de monitoreo colaborativo estos factores de éxito ya existan, pero con frecuencia no existen. Por ejemplo, una de las condiciones habilitantes cruciales es un entrenamiento apropiado y suficiente en actividades de monitoreo a los habitantes locales. En tanto que en un sitio bajo RPF se podría carecer de entrenamiento sólido en monitoreo colaborativo, es posible evaluar en el sitio el potencial de implementar los niveles apropiados de entrenamiento. Además, al describir los factores de éxito en todas las fases, la matriz puede servir como una herramienta de planeación que ayude a las organizaciones en la formulación de sus estrategias de monitoreo, ya sea para un sitio específico o en un esfuerzo de restauración con múltiples niveles. Al tener conocimiento de lo que debe alcanzarse en las fases posteriores, tanto los sitios de RPF como los sistemas multinivel de monitoreo pueden considerar con antelación factores de éxito necesarios y adelantarse a obstáculos potenciales.

\subsection{La escala de gobernanza}

La 'escala de gobernabilidad' a menudo se conceptualiza como un modelo lineal de actores organizados en jurisdicciones geográficas anidadas, es decir, desde arriba (nacional / global) hacia abajo (comunidad local o sitio de restauración). Si bien se trata de una simplificación, con fines prácticos y de claridad se han organizado los factores de éxito en este espectro: nacional / subnacional / local. En realidad, la escala de gobierno puede parecerse más a una red de actores que se influyen entre sí y comparten información (Pahl-Wostl 2009; Jedd y Bixler 2015), en la que algunos actores ocupan múltiples niveles, y los actores y las relaciones cambian con el tiempo de acuerdo a temas particulares (McDermott et al. 2010). Por lo tanto, algunos factores de éxito pueden ser relevantes a varios niveles simultáneamente, y como tal, es importante considerar el evaluar un factor de éxito a niveles diferentes a los que aquí se proponen. Por ejemplo, las mujeres en posiciones de liderazgo a nivel comunitario inspiran el compromiso de las mujeres en el monitoreo (Turreira-García et al. 2018) y también es un factor de éxito que podría evaluarse a nivel subregional y nacional. Como se mencionó anteriormente, esta matriz podría usarse como diagnóstico para un sitio de RPF específico o para un sistema de monitoreo multinivel que involucre múltiples sitios de RPF. En el primer caso, los factores de éxito en la categoría "local" obviamente serán más relevantes y, en el segundo caso, todos los niveles de gobernabilidad pueden ser aplicables.

\subsection{Motivar la participación local}

\begin{abstract}
Varios autores han identificado 'incentivos
y motivaciones locales' como los temas más importantes a abordar en el monitoreo colaborativo de la RPF (Saipothong et al. 2006; Fernandez-Gimenez et al. 2008; Le Tellier et al. 2009; Laake et al. 2013; Boissière et al. 2014; Danielsen et al. 2014; Bellfield et al. 2015; Villaseñor et al. 2016; Brites y Morsello 2017; Turreira-García et al. 2018). Por lo tanto, en este documento los factores de éxito relacionados con este tema tienen su propio encabezado.
\end{abstract}

\subsection{Asignando los puntajes}

La matriz de factores de éxito sirve como la 'tarjeta de puntuación' para las actividades de evaluación, que podría incluir una combinación de entrevistas, encuestas y/o talleres para calificar el estado de los factores de éxito. Hay varios enfoques posibles para la puntuación. Por ejemplo, el enfoque «semáforo» (Stanturf et al. 2015, p.53) es una técnica simple que brinda una representación visual del estatus de un factor de éxito al usar uno o tres valores ( $\mathrm{p}$. ej. rojo = no existe, no hay capacidad; amarillo = progresando o alguna capacidad; verde = existente, buena capacidad). De forma alternativa, puede ser útil asignar valores de la escala Likert ( 1 a 7 ) para representar más variaciones y brindar una base para cálculos básicos de los valores. Hay muchos otros métodos para ilustrar gráficamente la información que será comunicada a los actores interesados (véase Evans y Guariguata 2016). También sería conveniente acompañar los resultados con una explicación más refinada y contextualizada en un 
formato de informe. Junto a la columna llamada Puntuación en la matriz hay una columna llamada Intervención. Los usuarios pueden usar esta columna para anotar oportunidades o pasos concretos para mejorar los factores de éxito. La matriz no fue concebida para dar una calificación estática tipo "aprobado" o "reprobado", sino como una herramienta para reconocer fortalezas y fallas; si es factible que una intervenciòn mejore el estado de un factor de éxito, entonces puede anotarse.

La naturaleza de los factores de éxito dictará los métodos empleados para recolectar la información. En el apéndice 2 se enumeran varias guías de métodos. Estas guías dan instrucciones paso a paso de técnicas como el análisis de los actores interesados, mapeo participativo y puntuación de valores. Algunos de los factores de éxito pueden requerir consultas o entrevistas relativamente simples; otros pueden requerir un proceso de participación más completo para comprender en su totalidad el alcance de las condiciones y el contexto. Por ejemplo, comprender la naturaleza de las instituciones de gobierno y las relaciones entre las partes interesadas puede requerir la aplicación de técnicas tomadas del análisis de redes sociales (Newig et al. 2010; Devisscher et al. 2016; Fischer y Jasny 2017). Cada factor de éxito requerirá cierta consideración para determinar el método más apropiado, y factible, para evaluarlo. El tiempo y el costo son siempre preocupaciones centrales para una actividad de evaluación, y estas van a variar significativamente según la cantidad de sitios de restauración que se estén evaluando y sus características geográficas y de gestión. Estas consideraciones deben sopesarse cuando el usuario decide finalmente cómo evaluar un factor de éxito. 


\section{Matriz de factores de éxito esenciales}

A continuación se listan los factores de éxito esenciales, considerados centrales en cualquier iniciativa de monitoreo colaborativo de la RPF. Todos estos factores deben ser evaluados, sin embargo pueden ser necesarias algunas adaptaciones dependiendo del contexto. Por ejemplo, las partes interesadas relevantes en una RPF de un sitio que principalmente se ubica en tierras privadas habrán de variar considerablemente en comparación con los actores interesados en un proyecto de RPF en tierras comunitarias.

El apéndice 3 contiene una lista ampliada de factores de éxito adicionales que pueden ser relevantes en un sitio dado de RPF. Esta lista ampliada se puede utilizar para complementar los principales factores de éxito a continuación. Si bien es oportuno hacer una «lista de compra» personalizada, se debe tener cuidado de no seleccionar los factores que son los más fáciles de evaluar o los que tienen más probabilidades de obtener puntajes más altos; este tipo de sesgo de confirmación ${ }^{2}$ socavará la utilidad de la herramienta. Los enfoques tales como involucrar a un conjunto diverso de personas en la selección y análisis de los factores de éxito pueden ayudar a evitar el sesgo de confirmación.
2 El sesgo de confirmación ocurre cuando solo se seleccionan los factores de éxito con mayor probabilidad de tener alta calificación con el fin de confirmar una creencia que ya existía. 
Tabla 1. Matriz de factores de éxito esenciales.

\begin{tabular}{|c|c|c|}
\hline A. PREMONITOREO & \multicolumn{2}{|c|}{ Evaluación } \\
\hline $\begin{array}{l}\text { Factores de éxito en las fases iniciales de planeación de la RPF, previas al } \\
\text { monitoreo colaborativo }\end{array}$ & Puntaje & Intervención \\
\hline
\end{tabular}

\section{Sitio de RPF}

1. Se define toda el área geográfica que se espera que sea afectada por la RPF ${ }^{a}$

\section{Planeación de la RPF}

2. Las partes interesadas locales se involucran en decidir lo que constituye la RPF, qué constituye el éxito de la restauración y cuáles son los objetivos de la restauración

3. Los objetivos de restauración son simples, y las partes interesadas concuerdan generalmente con tales objetivos

4. Los objetivos de restauración se transforman en objetivos factibles y metas medibles ${ }^{\mathrm{b}}$

5. El monitoreo se considera esencial para el éxito de la restauración

6. Los objetivos y prioridades de todas las partes interesadas están incluidas en la planeación de la restauración, con estrategias específicas ${ }^{c}$ que involucren a mujeres y a grupos marginados en todas las fases

\section{Participantes locales}

7. La población local tiene derechos de acceso a la tierra y a los recursos naturales, y existen relativamente pocos conflictos sobre los derechos de acceso

8. El esfuerzo de la restauración está conformado por una amplia coalicióne de usuarios relevantes del paisaje involucrados de manera importante, ya sea que se trate de grupos marginados, castas, mujeres, jóvenes, líderes locales, pequeños propietarios, grandes propietarios, organizaciones no gubernamentales (ONG), compañías o gobiernos

9. Existen fuertes motivaciones intrínsecas para participar a nivel local en la restauración, y las partes interesadas locales perciben que hay un beneficio por su participación

10. Los participantes están involucrados en aspectos de repartición de beneficios o en actividades relacionadas con la restauración (p. ej. turismo, reforestación, etc.)

\section{Organización de la implementación local y del personal}

11. El personal de restauración está capacitado, motivado y bien remunerado para apoyar el monitoreo colaborativo

12. El personal de restauración reconoce que tiempo, negociación y entrenamiento son partes necesarias del proceso de monitoreo y se adhiere a un espíritu de aprendizaje, experimentación y participación

13. El personal de restauración posee una caja de herramientas diversa con técnicas de monitoreo relevantes y apropiadas a nivel local

14. El personal de restauración está motivado y tiene conocimientos sobre enfoques participativos que faciliten la recopilaciòn y anàlisis de datos, el intercambio de información y el aprendizaje

15. El monitoreo colaborativo está escrito en los planes de trabajo del personal de restauración, para que si hay un cambio de personal, el monitoreo continúe 
Tabla 1. Continuaciòn

\begin{tabular}{|c|c|c|c|}
\hline \multirow{2}{*}{\multicolumn{2}{|c|}{$\begin{array}{l}\text { A. PREMONITOREO (continuación) } \\
\text { Factores de éxito en las fases iniciales de planeación de la RPF, previas al } \\
\text { monitoreo colaborativo }\end{array}$}} & \multicolumn{2}{|c|}{ Evaluación } \\
\hline & & Puntaje & Intervención \\
\hline \multirow{5}{*}{ 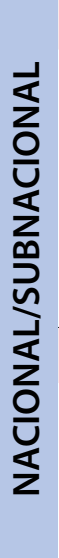 } & \multicolumn{3}{|l|}{ Gobernanza e instituciones } \\
\hline & $\begin{array}{l}\text { 16. Existe un compromiso concertado a largo plazo por parte de las partes } \\
\text { interesadas a nivel nacional y subnacional para establecer el sistema de } \\
\text { monitoreo colaborativo y llevarlo a cabo }\end{array}$ & & \\
\hline & $\begin{array}{l}\text { 17. Existen instituciones formales sólidas y cooperación entre instituciones } \\
\text { informales, toma de decisiones transparentes, distribución equitativa del } \\
\text { poder y bajos niveles de corrupción }\end{array}$ & & \\
\hline & \multicolumn{3}{|l|}{ Redes de aprendizaje } \\
\hline & $\begin{array}{l}\text { 18. Se identifica la "comunidad de práctica" (el grupo de personas } \\
\text { u organizaciones preocupadas por la restauración), la cual crea } \\
\text { oportunidades para intercambiar información e ideas regularmente a } \\
\text { través de organizaciones, sitios web, reuniones, talleres y conferencias }\end{array}$ & & \\
\hline
\end{tabular}

\section{B. PLANEACIÓN Y PREPARACIÓN}

Factores de éxito durante el diseño y planeación de actividades de monitoreo, incluyendo la elaboración de presupuesto, la capacitación para la población local y el personal de restauración, y la construcción de sistemas para compartir información

19. Las inversiones en capacitación, creación de capacidades y seguimiento se incluyen en los costos del monitoreo colaborativo

20. Los recursos se dedican al análisis de datos y actividades de aprendizaje social (reuniones, talleres, capacitaciones, excursiones) que apoyan ciclos de toma de decisiones y de gestión adaptativa

21. Se incluyen en el presupuesto los costos relacionados con el control de calidad, la gestión de datos y el almacenamiento de datos

22. Una parte específica del presupuesto de restauración se dedica a supervisar la duración del período de restauración, p. ej. 10\% del presupuesto total de restauración

\section{Planeación}

23. Los planes de monitoreo se hacen al principio de las etapas de planificación de la restauración, se ajustan a los objetivos de la restauración e involucran a una variedad de partes interesadas

24. Los indicadores de monitoreo están estrechamente alineados con los objetivos de gestión a corto, mediano y largo plazo

\section{Selección de preguntas e indicadores para el monitoreo}

25. El proceso de definición de preguntas/indicadores de monitoreo, incluido el uso de recursos naturales, el bienestar y otros, es colaborativo y enfatiza el aprendizaje mutuo

26. Los indicadores concuerdan con los objetivos de restauración

27. Los indicadores no son demasiado técnicos y no requieren una gran cantidad de conocimiento matemático

28. Los indicadores no consumen demasiado tiempo ni son excesivamente caros de monitorear, no son demasiados y son fáciles de interpretar

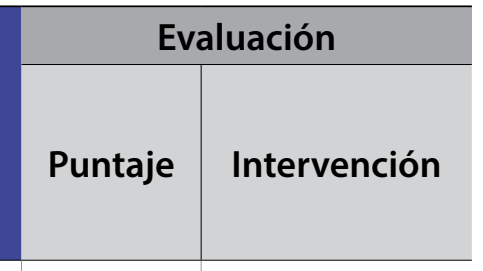

Evaluación

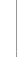


Tabla 1. Continuaciòn

B. PLANEACIÓN Y PREPARACIÓN (continuación)
Factores de éxito durante el diseño y planeación de actividades de
monitoreo, incluyendo la elaboración de presupuesto, la capacitación
para la población local y el personal de restauración, y la construcción de
sistemas para compartir información

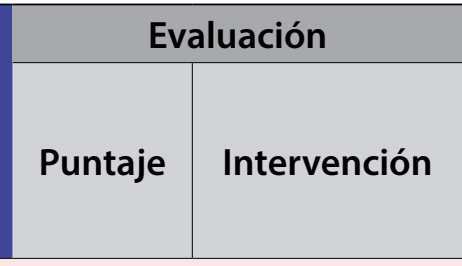

\section{Métodos y tecnología para la recolección de datos}

29. Las formas y los protocolos para la recolección de datos se diseñan en colaboración con monitores locales, investigadores y personal gubernamental; no se desarrollan de manera aislada

30. Los métodos y herramientas de recolección de datos están orientados a un procesamiento y análisis local y rápido sin cálculos complicados, y facilitan el intercambio con las partes interesadas en múltiples niveles y la aplicación en futuros esfuerzos de restauración.

\section{Capacitación y apoyo}

31. Se brinda capacitación con regularidad a los pobladores locales en el uso de las herramientas, formas y técnicas de recolección de datos, así como en la interpretación de datos para promover el entendimiento y la resolución de preguntas

32. La capacitación es simple y se adapta a la capacidad técnica de los participantes. ${ }^{f}$

\section{Incentivos y motivaciones locales}

33. Los participantes sienten que sus necesidades se toman en consideración en el sistema de monitoreo y que las actividades se concentran en atributos relevantes a los participantes en lugar de satisfacer criterios meramente científicos

34. Las necesidades y objetivos de las partes interesadas locales se someten a consideración desde el principio y concuerdan con las de científicos y gestores de recursos naturales

35. Los resultados del monitoreo, tanto del proyecto local como del programa en general, se comparten regularmente con el fin de motivar la participación

\section{Sistema de monitoreo de múltiples niveles}

36. Existe infraestructura para el registro, almacenaje y procesamiento de datos que permita ampliar la escala a nivel de un sistema nacional de monitoreo

37. Existen procedimientos estándar para que los procesos de monitoreo sean consistentes y transparentes, de forma que se pueda ampliar la escala a un sistema nacional de monitoreo

38. Existe un ente responsable de la organización y supervisión del monitoreo y de balancear las necesidades locales con las necesidades nacionales y globales 
Tabla 1. Continuaciòn

\section{IMPLEMENTACIÓN}

Factores de éxito durante las actividades de monitoreo, incluyendo

la recolección y análisis de datos, y el intercambio de información y el aprendizaje

\section{Evaluación}

\section{Puntaje Intervención}

\section{Recolección, análisis y aprendizaje de la información}

39. Los datos se recolectan desde el principio y luego a intervalos regulares

40. La información sobre el progreso logrado y los puntos a los que se desea llegar se representa de manera visual de forma que las partes interesadas la entiendan y se discuta de manera que los pobladores locales puedan interpretar y aplicar

41. Los pobladores locales se sienten cómodos al compartir sus impresiones y aprendizajes a pesar de diferencias de poder con los funcionarios, y sienten la facultad para efectuar cambios. ${ }^{g}$

42. Se organizan eventos de aprendizaje repetidamente como interacciones, reuniones y salidas de campo a los sitios de restauración, en los que participan las partes interesadas para estimular el aprendizaje y la adaptación, generar confianza, fomentar el respeto a opiniones diversas e incrementar la transparencia

a Podrían ser necesarios límites flexibles para incluir cambios o eventos imprevistos

b Medible puede significar evaluaciones simples, como del tipo "malo, satisfactorio, bueno"

c La participación de mujeres y grupos marginados es crucial, y debe abordarse de acuerdo al contexto

d Esto depende del contexto de tenencia de la tierra y de costumbres locales respecto al acceso

e La composición de esta coalición depende de los patrones de tenencia de la tierra y de derechos de uso. Además, los papeles y tipos de participación van a variar de acuerdo a los grupos

f Nótese que algunos proyectos implementan dos tipos de monitoreo: el monitoreo simple (hecho por los pobladores locales) y tareas más complejas (hechas por el personal del proyecto)

g Esto es un proceso que evoluciona a lo largo del tiempo 


\section{Glosario de conceptos y definiciones}

En esta sección se presentan conceptos clave y definiciones con el fin de establecer una base uniforme para entender los factores de éxito y emplear el diagnóstico.

Monitoreo - Es la recolección y análisis sistemático de la información para evaluar los cambios. El monitoreo es más que una sola evaluación; debe realizarse a intervalos regulares apropiados a la materia, eficiente en cuanto al costo y no demasiado pesado de realizar. La información se analiza y los resultados son evaluados y empleados en la toma de decisiones (Evans y Guariguata 2008).

Monitoreo y la RPF - Existe amplio acuerdo sobre lo fundamental que es el monitoreo para el éxito de los esfuerzos de restauración ecológica (Sociedad para la Restauración Ecológica Internacional, grupo de trabajo sobre ciencia y política 2004; Clewell y Aronson 2013; Sayer et al. 2013; Dey y Schweitzer 2014). En ausencia de un monitoreo del progreso y del cambio, es imposible evaluar si los esfuerzos de restauración son exitosos, están en el camino hacia el éxito o se están alejando de los objetivos de restauración (Holl y Cairns 2002). Además, el monitoreo genera la información que brinda las bases para el aprendizaje social y el manejo adaptativo, aspectos esenciales en el proceso de RPF (Le et al. 2012; Reed et al. 2016).

Monitoreo colaborativo y monitoreo participativo El monitoreo participativo se refiere a una línea de participación que va desde pobladores locales hasta investigadores que se dedican a la recolección y uso de datos para la toma de decisiones, principalmente a nivel local (Danielsen et al. 2009). El monitoreo colaborativo incluye estos tipos de actividades de monitoreo hecho por varias partes, y también comprende actores e interacciones de múltiples niveles y de escalas cruzadas, que se relacionan entre sí para compartir la información e influir en un cambio (Cash et al. 2006; Newig et al. 2010). En este diagnóstico se emplea de forma intencional el término monitoreo colaborativo en lugar del término monitoreo participativo más ampliamente usado, pues el monitoreo colaborativo reconoce la diversidad de actores e intereses, algunas veces en desacuerdo, presentes en el paisaje forestal y que están ligados a los esfuerzos de restauración, y porque se refiere a la importancia primordial del aprendizaje al interior de dichos grupos (Demeo et al. 2015; USDA Forest Service 2017).

Factores de éxito y restricciones - Los elementos que ayudan a una intervención determinada a lograr sus objetivos se denominan aquí 'factores de éxito'. Este término abarca condiciones contextuales y características estructurales, así como actores, actitudes y actividades. No sugiere causalidad, sino que alude a su papel para alcanzar el éxito (Hanson et al. 2015). Los factores que representan una barrera o un impacto negativo se denominan 'restricciones'. En este contexto, las restricciones pueden incluir ya sea impedimentos estructurales permanentes o barreras que pueden ser superadas gracias a una intervención (Moser y Ekstrom 2010; Devisscher et al. 2016).

Escala - En sí misma es una herramienta para comprender problemas de nuevas maneras; puede haber varios tipos: temporal, gubernamental, espacial, jurisdiccional, con base en conocimiento y con base en gestión, entre otros (Cash et al. 2006). Para ilustrar este punto, cada vez más se reconoce que la solución de problemas en la gestión de recursos naturales se ve obstaculizada por desajustes entre diferentes niveles al interior de una escala (p. ej. conflictos a escala de gobierno entre autoridades o normas locales y nacionales o subnacionales), o entre escalas (p. ej. los ciclos de financiamiento que definen el tiempo de los proyectos son mucho más cortos que el marco temporal para la restauración forestal) (Brown 2003; Cash et al. 2006; Gallemore et al. 2014). En ocasiones puede ser difícil conceptualizar la escala y puede llegar a sonar como un lenguaje especializado, de tal forma que es importante usarla como una herramienta que facilita el entender las ideas. 
Gobernanza, redes de aprendizaje y comunidades de práctica - La gobernanza de los recursos naturales se percibe cada vez más, no como una categorización jerárquica del 'gobierno' y de los 'gobernados', sino como una 'red de diferentes actores y redes que ayudan a formular e implementar la política ambiental y/o instrumentos políticos' (Pahl-Wostl 2009 p. 355).

La construcción de redes de aprendizaje y de comunidades de práctica que conectan a estos actores para que puedan aprender juntos, son elementos esenciales del contexto de gobernabilidad para el monitoreo colaborativo en la RPF (van Oosten et al. 2014). Es muy importante entender los contextos de gobernanza a nivel local, subnacional y nacional para identificar las capacidades y restricciones de un sistema de monitoreo colaborativo de múltiples niveles de la RPF (Pahl-Wostl 2009). Además, los problemas relacionados con la gobernanza con frecuencia constituyen el tropiezo más importante en cualquier sitio de RPF, incluyendo la fase de monitoreo (Mansourian et al. 2017). Para tal fin, es crucial desarrollar un sistema de monitoreo de varios niveles que represente la complejidad de la red de gobernanza. 


\section{Referencias}

Bellfield H, Sabogal D, Goodman L y Leggett M. 2015. Case study report: Community-Based Monitoring Systems for REDD+ in Guyana. Forests 6:133-56.

Besseau P, Graham $S$ y Christophersen T, eds. 2018. Restoring Forests and Landscapes: The Key to a Sustainable Future. Viena, Austria: Global Partnership on Forest and Landscape Restoration.

Boissière M, Bastide F, Basuki I, Pfund J y Boucard A. 2014. Can we make participatory NTFP monitoring work? Lessons learnt from the development of a multi-stakeholder system in Northern Laos. Biodiversity \& Conservation 23:149-70.

Borgatti SP. 2002. NetDraw Software for Network Visualization. Lexington, KY: Analytic Technologies. https://sites.google.com/site/ netdrawsoftware/home

Borgatti SP, Everett M y Freeman LC. 2002. Ucinet for Windows: software for social network analysis. Harvard, MA: Analytic Technologies. https:// sites.google.com/site/ucinetsoftware/home

Brites AD y Morsello C. 2017. Beliefs about the potential impacts of exploiting non-timber forest products predict voluntary participation in monitoring. Environmental Management 59:898-911.

Brown K. 2003. Integrating conservation and development: A case of institutional misfit. Frontiers in Ecology and the Environment 1:479-487.

Buckingham K, Ray S, Arakwiye B, Morales AG, Singh R, Maneerattana O, Wicaksono $\mathrm{S}$, Chrysolite H, Minnick A y Johnston L. 2018. Mapping Social Landscapes: A Guide to Identifying Networks, Priorities, and Values of Restoration Actors. Washington DC: Instituto de Recursos Mundiales.

Cash DW, Adger WN, Berkes F, Garden P, Lebel L, Olsson P, Pritchard L y Young O. 2006. Scale and cross-scale dynamics: Governance and information in a multilevel world. Ecology and Society 11: 8. http://www.jstor.org/ stable/26265993

Clewell AF y Aronson J. 2013. Ecological references. En: Clewell AF y Aronson J, eds. Ecological Restoration: Principles, Values, and Structure of an Emerging Profession. Washington DC: Island Press/Center for Resource Economics. 137-153. http://link.springer.com/ chapter/10.5822/978-1-59726-323-8_7

Danielsen F, Burgess ND, Balmford A, Donald PF, Funder M, Jones JPG, Alviola P, Balete DS, Blomley T Brashares J et al. 2009. Local participation in natural resource monitoring: A characterization of approaches. Conservation Biology 23:31-42.

Danielsen F, Jensen PM, Burgess ND, Altamirano $\mathrm{R}$, Alviola PA, Andrianandrasana $\mathrm{H}$, Brashares JS, Burton AC, Coronado I, Corpuz N et al. 2014. A multicountry assessment of tropical resource monitoring by local communities. BioScience 64:236-251.

Demeo T, Markus A, Bormann B y Leingang J. 2015. Tracking progress: The monitoring process used in collaborative forest landscape restoration projects in the Pacific Northwest Region. Working Paper Number 54, Ecosystem Workforce Program. Portland, OR: University of Oregon.

Devisscher T, Vignola R, Coll Besa M, Cronenbold R, Pacheco N, Schillinger R, Canedi V, Sandoval C, Gonzalez D y Leclerc G. 2016. Understanding the socio-institutional context to support adaptation for future water security in forest landscapes. Ecology and Society 21:48. https://www.ecologyandsociety.org/ vol21/iss4/art48/

Dey DC y Schweitzer CJ. 2014. Restoration for the future: Endpoints, targets, and indicators of progress and success. Journal of Sustainable Forestry 33:S43-S65.

Edwards PM, Shaloum G y Bedell D. 2017. A unique role for citizen science in ecological restoration: A case study in streams. Restoration Ecology 26:29-35. 
Evans K y Guariguata MR. 2016. Success from the ground up: Participatory monitoring and forest restoration. Bogor, Indonesia: Centro para la Investigación Forestal Internacional (CIFOR). http://www.cifor.org/library/6284/successfrom-the-ground-up-participatory-monitoringand-forest-restoration/

Evans K y Guariguata MR. 2008. Participatory Monitoring in Tropical Forest Management: A Review of Tools, Concepts and Lessons Learned. Bogor, Indonesia: Centro para la Investigación Forestal Internacional (CIFOR). http:// www.cifor.org/publications/pdf_files/Books/ BGuariguata0801.pdf

Evans K, Guariguata MR y Brancalion PHS. 2018. Participatory monitoring to connect local and global priorities for forest restoration. Conservation Biology 32:525-34.

Evans K, de Jong W, Cronkleton P, Sheil D, Lynam T, Kusumanto Y y Colfer C. 2006. Guide to Participatory Tools for Forest Communities. Bogor, Indonesia: Centro para la Investigación Forestal Internacional (CIFOR). http://www. cifor.cgiar.org/Publications

Fernandez-Gimenez ME, Ballard HL y Sturtevant VE. 2008. Adaptive management and social learning in collaborative and community-based monitoring: A study of five community-based forestry organizations in the western USA. Ecology and Society 13:4.

Fischer AP y Jasny L. 2017. Capacity to adapt to environmental change: Evidence from a network of organizations concerned with increasing wildfire risk. Ecology and Society 22. http://www.jstor.org/stable/26270065.

Gallemore C, Rut HP y Moeliono M. 2014. Discursive barriers and cross-scale forest governance in Central Kalimantan, Indonesia. Ecology and Society 19. https://www. ecologyandsociety.org/vol19/iss2/art18/

Hanson C, Buckingham K, DeWitt $S$ y Laestadius L. 2015. The Restoration Diagnostic: A Method for Developing Forest Landscape Restoration Strategies by Rapidly Assessing the Status of Key Success Factors. Washington DC: Instituto de Recursos Mundiales. http://www.wri.org/ publication/restoration-diagnostic

Holl KD y Cairns J. 2002. Monitoring and appraisal. En: Holl KD y Cairns J, eds. Handbook of Ecological Restoration. Cambridge, UK: Cambridge University Press. 411-32.

Jedd T y Bixler RP. 2015. Accountability in networked governance: Learning from a case of landscape-scale forest conservation.
Environmental Policy and Governance 25:17287.

Laake P van, Skutsch M y McCall MK. 2013. Community forest monitoring. En: Achard F, ed. Reducing Greenhouse Gas Emissions from Deforestation and Degradation in Developing Countries: A Sourcebook of Methods and Procedures for Monitoring Measuring and Reporting. Wageningen, The Netherlands: Global Observation of Forest Cover and Land Dynamics GOFC-Gold. 187-202. http:// www.gofcgold.wur.nl/redd/index.php

Le HD, Smith C, Herbohn J y Harrison S. 2012. More than just trees: Assessing reforestation success in tropical developing countries. Journal of Rural Studies 28:5-19.

Le Tellier V, Carrasco A y Asquith N. 2009. Attempts to determine the effects of forest cover on stream flow by direct hydrological measurements in Los Negros, Bolivia. Forest Ecology and Management 258:1881-8.

Mansourian S, Dudley N y Vallauri D. 2017. Forest landscape restoration: Progress in the last decade and remaining challenges. Ecological Restoration 35:281-8.

Mansourian S, Vallauri D y Dudley N. 2005. Forest Restoration in Landscapes: Beyond Planting Trees. Berlin: Springer Science \& Business Media.

McDermott CL, Humphreys D, Wildburger C, Wood P, Marfo E, Pacheco P y Yasmi Y. 2010. Mapping the core actors and issues defining international forest governance. IUFRO World Series 28:19-36.

Moser SC y Ekstrom JA. 2010. A framework to diagnose barriers to climate change adaptation. Proceedings of the National Academy of Sciences 107:22026-31.

Newig J, Günther D y Pahl-Wostl C. 2010. Synapses in the Network: Learning in governance networks in the context of environmental management. Ecology and Society 15. https://www.ecologyandsociety.org/ vol15/iss4/art24/

Pahl-Wostl C. 2009. A conceptual framework for analysing adaptive capacity and multi-level learning processes in resource governance regimes. Global Environmental Change 19:354-65.

Reed J, Van Vianen J, Deakin EL, Barlow J y Sunderland T. 2016. Integrated landscape approaches to managing social and environmental issues in the tropics: Learning from the past to guide the future. Global Change Biology 22(7): 2540-54. 
Saipothong P, Preechapanya P, Promduang

T, Kaewpoka N y Thomas DE. 2006.

Community-based watershed monitoring and management in Northern Thailand. Mountain Research and Development 26:289-91.

Sayer J, Sunderland T, Ghazoul J, Pfund J-L, Sheil D, Meijaard E, Venter M, Boedhihartono AK, Day M, Garcia C et al. 2013. Ten principles for a landscape approach to reconciling agriculture, conservation, and other competing land uses. Proceedings of the National Academy of Sciences 110:8349-56.

Schiffer E. 2018, September 24. Net-Map. https:// netmap.wordpress.com/about/

Schultz CA, Coelho DL y Beam RD. 2014. Design and governance of multiparty monitoring under the USDA Forest Service's Collaborative Forest Landscape Restoration Program. Journal of Forestry 112:198-206.

Sociedad para la Restauración Ecológica Internacional, grupo de trabajo sobre ciencia y política. 2004. The SER International Primer on Ecological Restoration. www.ser.org \& Tucson, AZ: Society for Ecological Restoration International. http://www.ser.org/resources/ resources-detail-view/ser-international-primeron-ecological-restoration\#8

Stanturf JA, Kant P, Lillesø J-PB, Mansourian $S$, Kleine M, Graudal L y Madsen P.
2015. Forest Landscape Restoration as a Key Component of Climate Change Mitigation and Adaptation. Volume 34, IUFRO World Series. Vienna, Austria: IUFRO. http://www. iufro.org/publications/series/world-series/ article/2015/12/01/world-series-vol-34-forestlandscape-restoration-as-a-key-component-ofclimate-change-mitigation/

Turreira-García N, Meilby H, Brofeldt S, Argyriou D y Theilade I. 2018. Who wants to save the forest? Characterizing communityled monitoring in Prey Lang, Cambodia. Environmental Management 61:1019-30.

USDA Forest Service. 2017. Expert Consultation on Monitoring Forest Landscape Restoration Workshop Report. Washington DC: United States Forest Service Office of International Programs.

van Oosten C, Gunarso P, Koesoetjahjo I y Wiersum F. 2014. Governing forest landscape restoration: Cases from Indonesia. Forests 5:1143-62.

Villaseñor E, Porter-Bolland L, Escobar F, Guariguata MR y Moreno-Casasola P. 2016. Characteristics of participatory monitoring projects and their relationship to decisionmaking in biological resource management: A review. Biodiversity and Conservation 25:2001-19. 


\section{Apéndice 1. Métodos}

En esta sección se describe el proceso de desarrollo del diagnóstico, específicamente la identificación de los factores de éxito. En el año 2018 CIFOR realizó un estudio para identificar tanto el conocimiento como las lecciones aprendidas ya existentes con el fin de ampliar y profundizar el entendimiento del potencial del monitoreo colaborativo, y la producción de varias publicaciones (p. ej. Evans y Guariguata 2016; Evans et al. 2018). La visión del monitoreo como un proceso de arriba hacia abajo probablemente no generará la información, la toma de decisiones adaptativa y la aceptación local, aspectos que son cruciales para lograr los múltiples objetivos biofísicos y socioeconómicos de la intervención de RPF (Fernandez-Gimenez et al. 2008; Schultz et al. 2014; Demeo et al. 2015). Se realizó una búsqueda de literatura revisada por pares con el fin de identificar casos de monitoreo colaborativo en RPF específicamente, y en general en la gestión de recursos naturales. Las bases de datos en las que se busó la información incluyeron el Science Citation Index Expanded, Social Sciences Citation Index, Environment Complete, AGRIS, GreenFILE, ScienceDirect, OAIster, MEDLINE, la Biblioteca Digital en Red de Tesis y Disertaciones, publicaciones en JSTOR, GEORef, BioOne y SciTech. Las combinaciones de palabras relevantes incluyeron restauración del paisaje forestal, restauración forestal, restauración, rehabilitación, restauración del ecosistema, monitoreo colaborativo, monitoreo participativo, monitoreo con base comunitaria, monitoreo local, monitoreo comunitario, regeneración natural manejada por agricultores, agroforestería, bosque, paisaje, cuenca hídrica, erosión del suelo, servicios del ecosistema, gobernanza, gobernanza en red, redes de gobernanza, escala, análisis de redes sociales, sistemas socioecológicos.

Del resultado de estas búsquedas, y de las bibliografías de los artículos encontrados en las búsquedas, se identificaron y revisaron aproximadamente 80 artículos relevantes. Se identificaron los factores de éxito y las restricciones que impactan el monitoreo colaborativo mediante al empleo de las siguientes preguntas:

- ¿Cuáles fueron las condiciones biofísicas, sociales e institucionales del sitio o los casos descritos que contribuyeron a (o restringieron) un monitoreo colaborativo exitoso?

- ¿Quiénes participaron (pobladores locales, actores de nivel subnacional, nacional o global, instituciones, líderes carismáticos, defensores), cuáles fueron sus roles y cuáles fueron las características de su participación exitosa (o no exitosa)?

- ¿Cuáles fueron los otros factores que contribuyeron (o restringieron) el éxito, incluyendo, pero no limitado a estos, estructuras de gobierno, organizaciones, redes, recursos, tecnología, eventos, financiamiento, flujos de información y dinámicas de escala?

Los factores de éxito incluidos en la matriz fueron los más relevantes con base en la frecuencia y énfasis con que aparecen en la literatura. Los resultados se agruparon $y$ sintetizaron en una serie de enunciados que pueden evaluarse a través de preguntas, entrevistas o talleres. Los factores de éxito fueron el producto de una evaluación independiente llevada a cabo por 20 expertos globales, quienes los priorizaron en base a su utilidad, relevancia e importancia. La tabla 1 presenta los 42 factores de éxito con el mayor valor de priorización, considerados como esenciales. La lista completa se encuentra en el apéndice 3.

Como se mencionó anteriormente, a la fecha no existen redes de monitoreo colaborativo de escala ajustable, que puedan brindar el tipo de intercambio de información y aprendizaje a 
múltiples escalas, las cuales consideramos necesarias para el éxito de una agenda de RPF de nivel nacional e internacional. Por lo tanto, el presente documento no pretende ser un análisis cuantitativo de casos publicados; la muestra disponible no es lo suficientemente grande o descrita consistentemente para hacer comparaciones confiables. No obstante, existen varios ejemplos $(\mathrm{n} \approx 80)$ de monitoreo colaborativo de restauración forestal, gobernanza policéntrica, redes de aprendizaje y otros temas relacionados que contribuyen de forma colectiva a identificar los factores de éxito necesarios y las restricciones y que permiten elaborar este diagnóstico de monitoreo colaborativo. 


\section{Apéndice 2. Enfoques de puntuación y recursos de evaluación}

Varios de los factores de éxito pueden evaluarse empleando información común obtenida mediante entrevistas, grupos focales o encuestas, los cuales a la vez son herramientas y recursos de evaluación, orientados a la recolección de información en un ambiente grupal. A menudo, la información obtenida puede llevar a una comprensión más rica de las complejidades de los factores de éxito. Varios de estos recursos de evaluación y herramientas se indican a continuación. Cabe anotar que se debe tener cuidado en obtener el consentimiento previo e informado de todos los participantes.

Guía de herramientas participativas en comunidades forestales (Evans et al. 2006) - Esta guía describe varias herramientas que pueden utilizarse para obtener información para la evaluación. Las herramientas para evaluar las relaciones de las partes interesadas incluyen la matriz Principales Involucrados (matriz Who Counts, en inglés) diagramas de Venn, puntuación con guijarros y la herramienta denominada Cuatro R. Otras herramientas como el mapeo participativo, pueden obtener percepciones sobre paisajes, en tanto que otras herramientas pueden obtener perspectivas sobre un tema, como la valoración basada en el discurso, la puntuación de guijarros y los diagramas radiales. http://www.cifor.org/publications/pdf_files/ Books/BKristen0601.pdf

Análisis de redes sociales (ARS) - El ARS ha sido empleado en varios contextos para identificar los actores clave relacionados con un tema específico, determinar su escala espacial de influencia y cuantificar sus relaciones e influencia en cada uno de ellos, así como las barreras que impiden su éxito. El artículo titulado "Comprensión del contexto socioinstitucional de apoyo a la adaptación para una seguridad hídrica futura en paisajes forestales" (Devisscher et al. 2016) describe la aplicación del ARS en comunidades forestales y los métodos empleados, incluyendo el mapeo participativo social, entrevistas semiestructuradas y talleres de validación. El proceso es intensivo en cuanto a tiempo, pero podría modificarse. Identifica barreras críticas y cuellos de botella (falta de conocimiento, de confianza y de apoyo institucional) relacionados con el tema del agua.

Otro ejemplo relevante de ARS se puede encontrar en la publicación de Fischer y Jasny 2017, "Capacidad de adaptación al cambio del medio ambiente: Evidencia de una red de organizaciones preocupadas del creciente riesgo para la vida silvestre". Los métodos son más simples y se emplean entrevistas (algunas en persona, otras por correo electrónico) mediante un enfoque de bola de nieve. Para visualizar las redes y analizar sus valores métricos, los dos ejemplos emplearon los programas de software Netdraw (Borgatti 2002) y UCInet (Borgatti et al. 2002).

Schiffer (2018) desarrolló un método simplificado e interactivo sin uso de computadora que puede emplearse con actores interesados de todos los niveles de educación denominado Net-Map. Dicho método también es utilizado por el Instituto de Recursos Mundiales en el estudio Mapeo de paisajes sociales (Buckingham et al. 2018) (véase a continuación).

Mapeo de paisajes sociales: Una guía para la identificación de redes, prioridades y valores de actores en la restauración (Buckingham et al. 2018). En este estudio se adaptaron varios enfoques analíticos para entender cómo se organizan las personas en el terreno. Emplea dos enfoques, ARS y cuestionarios de prioridades y valores, con el fin de entender las relaciones, papeles e influencia de actores en el paisaje.

El diagnóstico de la restauración: Un método para desarrollar estrategias de restauración del paisaje forestal mediante la evaluación rápida del estado de varios factores clave de éxito (Hanson et al. 2015). En el documento, los autores crearon los criterios para una RPF exitosa mediante el análisis de casos históricos, y desarrollaron una herramienta de diagnóstico empleada en talleres y entrevistas con actores clave para luego ser probada en el campo. Parece ser un producto sólido y completo. 


\section{Apéndice 3. Lista ampliada de factores de éxito}

A continuación se incluye la lista completa de factores de éxito que se identificaron. Esta lista ampliada incluye los factores de éxito 'esenciales' que aparecen en el texto principal del documento, sumados a factores de éxito opcionales. Como se mencionaba en el texto principal, en tanto que se espera que se elabore una 'lista de compras', se debe tener cuidado de no escoger solo los factores más fáciles de evaluar o los que tienen mayor probabilidad de arrojar puntajes altos; este tipo de confirmación sesgada socavará la utilidad de la herramienta. Los enfoques que involucran un conjunto diverso de personas en la selección y análisis de factores de éxito pueden evitar la confirmación sesgada.

\begin{tabular}{|c|c|c|c|}
\hline A. PREMONITOREO & \multicolumn{2}{|c|}{ Evaluación } & \multirow[b]{2}{*}{ Referencia } \\
\hline $\begin{array}{l}\text { Factores de éxito en las fases iniciales de planeación de la RPF, } \\
\text { previas al monitoreo colaborativo }\end{array}$ & Puntaje & Intervención & \\
\hline
\end{tabular}

\section{Sitio de RPF}

1. Se define toda el área geográfica que se espera que sea afectada por la RPF

2. Existe acceso físico seguro hacia y dentro de los sitios de restauración en los momentos en los que se requiere hacer trabajo de restauración

3. Existe disponibilidad de mapas temáticos/biofísicos

\section{Planeación de la RPF}

4. Se entienden los impactos de la pérdida de bosque, y se han definido las causas de la deforestación

5. Las partes interesadas a nivel local se involucran en decidir qué constituye la RPF, qué constituye el éxito de la restauración y cuáles son los objetivos de la restauración

6. Los objetivos de restauración son simples, y las partes interesadas concuerdan generalmente con tales objetivos

7. Los objetivos de restauración se transforman en objetivos factibles y metas medibles

8. El personal a cargo de la restauración en el sitio ha adoptado enfoques adaptativos como la adaptación de objetivos y metas a lo largo del tiempo, para ajustar eventos imprevistos

9. El monitoreo se considera esencial para el éxito de la restauración

10. Los objetivos y prioridades de todas las partes interesadas están incluidas en la planeación de la restauración, con estrategias específicas que involucren a mujeres y a grupos marginados en todas las fases

11. El monitoreo colaborativo no se ve como una forma de ahorrar dinero que traslada el costo de las actividades de monitoreo a la población local 


\section{A. PREMONITOREO (continuación) \\ Evaluación \\ Factores de éxito en las fases iniciales de planeación de la RPF, previas al monitoreo colaborativo \\ Puntaje Intervención \\ Referencia}

\section{Participantes locales}

12. La población local conoce a fondo su bosque

13. La población local tiene derechos de acceso a la tierra y los recursos naturales

14. El esfuerzo de la restauración está conformado por una amplia coalición de usuarios relevantes del paisaje involucrados de manera importante, ya sea que se trate de grupos marginados, castas, mujeres, jóvenes, líderes locales, pequeños propietarios, grandes propietarios, organizaciones no gubernamentales (ONG), compañías o gobiernos

15. Existen fuertes motivaciones intrínsecas a nivel local para participar en la restauración, y las partes interesadas locales perciben que hay un beneficio por su participación

16. Los participantes están involucrados en aspectos de repartición de beneficios o en actividades relacionadas con la restauración (p. ej. turismo, reforestación, etc.)

17. Las partes interesadas no se han cansado por la presencia constante de actividades por agencias de desarrollo

\section{Implementación de la RPF, organización y características del personal}

18. El personal de restauración está capacitado, motivado y bien remunerado para apoyar el monitoreo colaborativo

19. Existe un equipo multidisciplinario cuyos miembros colaboran juntos de manera efectiva: por ejemplo, ecólogos, agrónomos, científicos sociales y pobladores locales con experiencia en plantaciones, cultivos y suelos en el lugar, así como en facilitación, análisis social y resolución de conflictos

20. Existe la capacidad para apoyar el entrenamiento de un gran número de participantes en la recolección de datos, el uso de herramientas, el análisis de datos, y en la interpretación y uso de datos para el intercambio de información

21. El personal de restauración reconoce que tiempo, negociación y entrenamiento son partes necesarias del proceso de monitoreo y se adhiere a un espíritu de aprendizaje, experimentación y participación

22. Las diferencias de poder y de conocimiento entre el personal y los pobladores locales son reconocidas y abordadas, y el personal es respetuoso, flexible y humilde

23. El personal para la restauración cuenta con un amplio repertorio de técnicas de monitoreo potencialmente relevantes y apropiadas a nivel local

24. El personal de restauración está motivado y tiene conocimientos sobre enfoques participativos que faciliten la recopilación y el análisis de datos, el intercambio de información y el aprendizaje

25. El monitoreo colaborativo está escrito en los planes de trabajo del personal de restauración, para que si hay un cambio de personal, el monitoreo continúe

26. Existe acceso seguro y conveniente a los recursos técnicos apropiados como dispositivos digitales, computadoras, software, imágenes de satélite y conexión a internet 


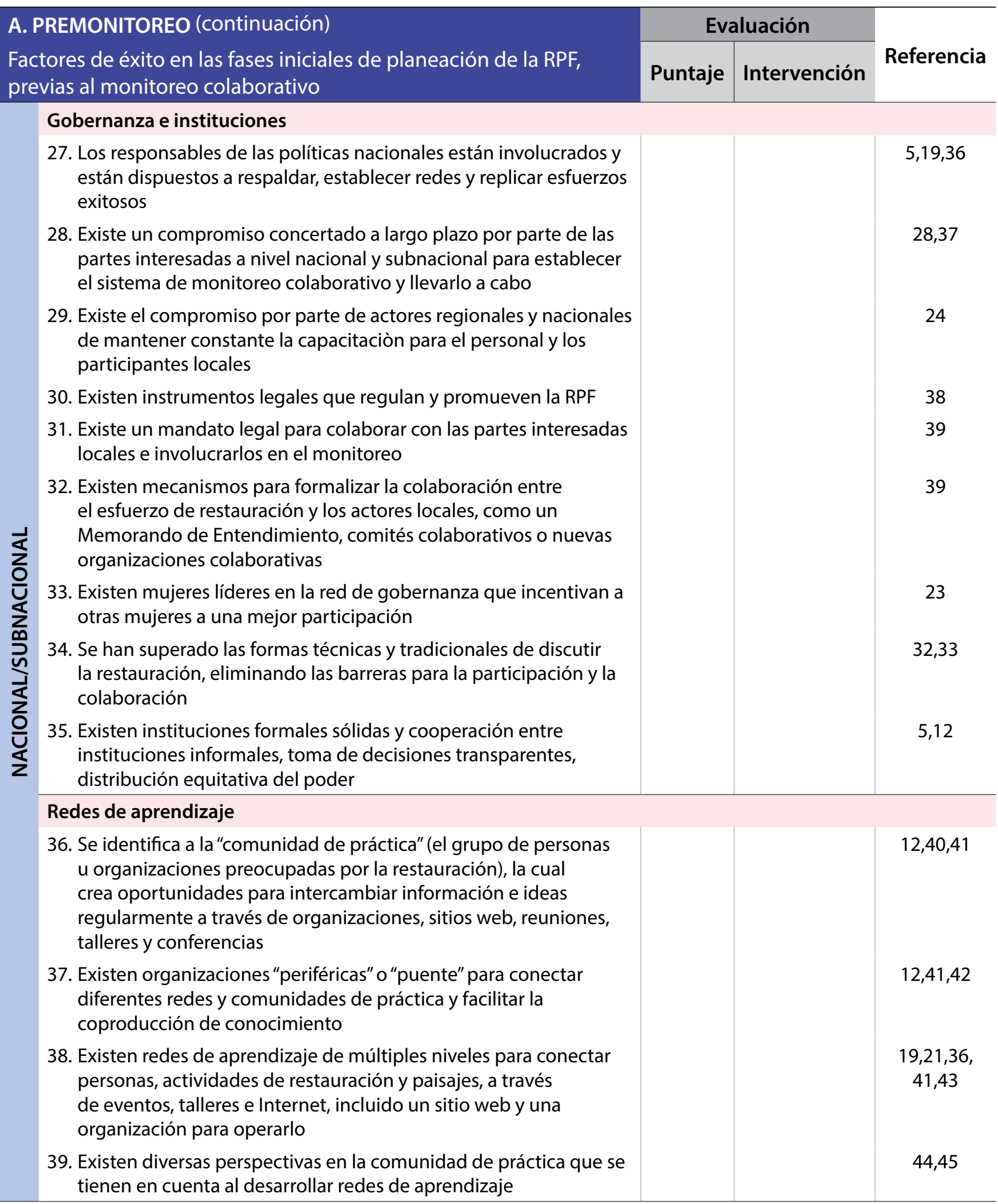




\section{B. PLANEACIÓN Y PREPARACIÓN}

Factores de éxito durante el diseño y planeación de actividades de monitoreo, incluyendo la elaboración de presupuesto, la capacitación para la población local y el personal de restauración, y la construcción de sistemas para compartir información

\section{Evaluación}

Puntaje Intervención

\section{Elaboración de presupuesto}

40. Las inversiones en capacitación, creación de capacidades y seguimiento se incluyen en los costos del monitoreo colaborativo

41. Los recursos se dedican al análisis de datos y actividades de aprendizaje social (reuniones, talleres, capacitaciones, excursiones) que apoyan ciclos de toma de decisiones y de gestión adaptativa

42. Se incluyen en el presupuesto los costos relacionados con el control de calidad, la gestión y el almacenamiento de datos

43. Una parte específica del presupuesto de restauración se destina a la supervisión de la duración del período de restauración, p. ej. $10 \%$ del presupuesto total de restauración

44. El presupuesto para la restauración incluye recursos destinados al intercambio de conocimientos para guiar la acción efectiva y la gestión adaptativa

\section{Planeación}

45. Los planes de monitoreo se hacen al principio de las etapas de planificación de la restauración, se ajustan a los objetivos de la restauración e involucran a una variedad de partes interesadas

46. Hay umbrales de acción, o sistemas de alerta temprana, en caso de que las cosas no funcionen, así como hitos o puntos de activación para juzgar el progreso

47. Existe un espíritu de experimentación que se concentra en pequeños ejercicios que se pueden cambiar en base al aprendizaje

48. Los indicadores de monitoreo están estrechamente alineados con los objetivos de gestión a corto, mediano y largo plazo

\section{Selección de preguntas e indicadores para el monitoreo}

49. El proceso de definición de preguntas/indicadores de monitoreo, incluido el uso de recursos naturales, el bienestar y otros, es colaborativo y enfatiza el aprendizaje mutuo

50. El marco de monitoreo incluye tanto indicadores de éxito como factores que contribuyen al éxito

51. Existe ayuda externa para desarrollar los indicadores

52. Se ha planeado hacer varias reuniones o talleres para definir indicadores mediante un proceso de búsqueda sistemática y de reducción del número de indicadores, basados en criterios

53. Los indicadores corresponden con las metas de restauración

54. Existe un proceso para identificar los hitos y los puntos de activación después de aplicar los indicadores

55. Existen estrategias especiales para involucrar a mujeres y grupos marginados, que incluyen la organización de grupos mixtos/ separados, esfuerzos de divulgación especiales para asegurar que los recursos técnicos y de capacitación lleguen a esos grupos, y la participación prioritaria en algunos protocolos de monitoreo

56. Los indicadores no son demasiado técnicos y no requieren una gran cantidad de conocimiento matemático 
B. PLANEACIÓN Y PREPARACIÓN (continuación)

Factores de éxito durante el diseño y planeación de actividades

de monitoreo, incluyendo la elaboración de presupuesto, la

capacitación para la población local y el personal de restauración, y

la construcción de sistemas para compartir información

\begin{tabular}{c|l}
\multicolumn{2}{c}{ Evaluación } \\
\hline Puntaje & Intervención Referencia \\
\hline
\end{tabular}

$25,27-29,54$

58. Los indicadores son decididos localmente

\section{Métodos y tecnología para la recolección de datos}

59. Las formas y los protocolos para la recolección de datos se diseñan en colaboración con monitores locales, investigadores y personal gubernamental; no se desarrollan de manera aislada

60. El nivel apropiado de precisión, no necesariamente el más riguroso científicamente, se utiliza para determinar los métodos de recolección de datos

61. Los métodos y herramientas de recolección de datos están orientados a un procesamiento y análisis local y rápido, sin cálculos complicados, y facilitan el intercambio con las partes interesadas en múltiples niveles y la aplicación en futuros esfuerzos de restauración

62. Existe disponibilidad de dispositivos móviles con copias de seguridad en papel para el monitoreo

63. Existe un sistema de verificación cruzada que incluye triangulación con expertos, controles aleatorios o análisis estadísticos para identificar anomalías y garantizar la fiabilidad, precisión e integridad de los datos y para evitar la fabricación de estos

64. Existen criterios básicos para evaluar la integridad y consistencia de los registros

65. Se han identificado los datos existentes (como la información social ya recolectada por el gobierno) de forma que puedan ser utilizados

66. Si se utilizan, las aplicaciones de ingreso de datos están dirigidas a personas con un nivel de alfabetización limitado y existe el compromiso de proporcionar conocimientos y apoyo externos continuos

67. Los dispositivos digitales no son percibidos como una panacea y solo se usan si existe una base sólida de preguntas, objetivos y enfoques definidos en colaboración

\section{Capacitación y apoyo}

68. Regularmente, se brinda capacitación a los pobladores locales en el uso de las herramientas, formas y técnicas de recolección de datos, así como en la interpretación de datos para promover el entendimiento y resolver dudas

69. La capacitación es simple y se adapta a la capacidad técnica de los participantes

70. Tanto la asistencia técnica como las capacidades son suficientes para asegurar la validez y credibilidad científica del monitoreo

71. Existe la disponibilidad de recursos técnicos adecuados como computadoras, software, imágenes de satélite y conexión a Internet, para comunicar resultados a las partes interesadas

72. Existe personal regional capacitado y bien remunerado para 


\section{B. PLANEACIÓN Y PREPARACIÓN (continuación)}

Factores de éxito durante el diseño y planeación de actividades de monitoreo, incluyendo la elaboración de presupuesto, la

capacitación para la población local y el personal de restauración, y

la construcción de sistemas para compartir información

\section{Incentivos y motivaciones locales}

73. Los beneficios o los incentivos económicos se encuentran disponibles para los participantes locales, son competitivos con otros medios de vida y se proporcionan al menos una vez al año (no al final de una fase plurianual)

74. Se siguen varias estrategias que promueven la participación, proporcionando incentivos y eliminando barreras que impiden la participación

75. Los participantes sienten que sus necesidades se consideran en el sistema de monitoreo, y las actividades se centran en atributos que son relevantes para ellos en lugar de cumplir con criterios enteramente científicos

76. La necesidad de información y los objetivos de las partes interesadas locales se consideran desde el principio y concuerdan con las de los investigadores y administradores de recursos naturales

77. Para mantener el interés, los participantes locales recolectan datos o se involucran en actividades relacionadas de manera regular

78. El monitoreo genera información que la población local puede usar en sus propias actividades productivas, como la agricultura y la caza

79. Los resultados del monitoreo, tanto del proyecto local como del proyecto en general, se comparten regularmente para motivar la participación

80. Los beneficios monetarios no están vinculados a los resultados del monitoreo, para evitar incentivar la falsificación, la manipulación o la fabricación de datos

81. Hay confianza en las autoridades y las personas no desconfían de brindar información

\section{Sistema de monitoreo de múltiples niveles}

82. Existe infraestructura para el registro, almacenaje y procesamiento de datos que permita ampliar la escala a nivel de un sistema nacional de monitoreo

83. Existen procedimientos estándar para que los procesos de monitoreo sean consistentes y transparentes de forma que se pueda ampliar la escala a un sistema nacional de monitoreo

84. Existe un ente de gestión que se encarga de organizar y supervisar y el equilibrio de las necesidades locales con las necesidades nacionales y mundiales

85. Existe un marco nacional de monitoreo que integra las necesidades locales con las demandas internacionales

86. Existe un marco nacional de monitoreo que proporciona un pequeño conjunto de indicadores nacionales comunes, y un banco de indicadores del cual los sitios pueden seleccionar

\begin{tabular}{c|l}
\multicolumn{2}{c}{ Evaluación } \\
\hline \multirow{2}{*}{ Puntaje } & Intervención
\end{tabular}

$7,17,29,34$

$23,37,65$

$18,27,51,59$

29

34

66

28,67

18,62 
B. PLANEACIÓN Y PREPARACIÓN (continuación)

Factores de éxito durante el diseño y planeación de actividades de monitoreo, incluyendo la elaboración de presupuesto, la

capacitación para la población local y el personal de restauración, y

la construcción de sistemas para compartir información

Sistema de monitoreo de múltiples niveles (continuación)

87. Existe algo más que un protocolo de monitoreo; hay un sistema de monitoreo que puede implementarse de manera realista y respaldar la recopilación, agregación, análisis, adaptación y aprendizaje de datos

88. El sistema de monitoreo incluye la capacidad de aprender y adaptarse

89. Los responsables del diseño de políticas/toma de decisiones reconocen los sistemas de monitoreo local y se basan en los mismos sistemas cuando es posible

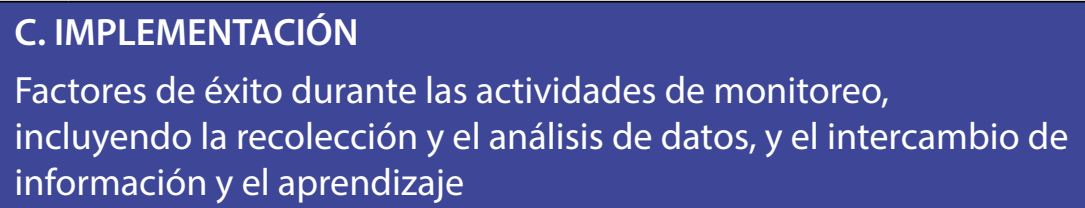

\begin{tabular}{|l|l|}
\hline \multicolumn{2}{|c|}{ Evaluación } \\
\hline Puntaje & Intervención \\
\hline
\end{tabular}

Referencia

\section{Recolección, manejo y análisis de información}

90. Los datos se recolectan desde el principio y luego a intervalos regulares

91. Se recolectan datos sobre el proceso mismo de monitoreo: sesiones de trabajo, tratamientos y costos

92. La información, resultado de las actividades de monitoreo, se registra regularmente en una base de datos de conocimientos tradicionales y en una base de datos GIS

93. Existe un protocolo de propiedad y uso compartido de datos que define quién es el propietario de la información y cómo se comparte, y existe soporte profesional para la gestión, el almacenamiento y el análisis de los datos

94. Los profesionales y los miembros de la comunidad participan conjuntamente en el análisis de datos y en el intercambio de información, que utilizan para la toma de decisiones y para identificar inconsistencias

95. La información obtenida durante el monitoreo se analiza con frecuencia, luego de la recopilación de pequeñas cantidades de datos, y no al final de las actividades de restauración

96. Los pobladores locales entienden con claridad cómo interpretar y usar los datos provenientes de herramientas desarrolladas por investigadores

\section{Aprendizaje, redes de aprendizaje e intercambio de información}

97. Los monitores se reúnen con regularidad para discutir los resultados e intercambiar información y datos

98. Se presenta la información sobre el progreso y las metas deseadas de forma que las partes interesadas la entiendan visualmente, y se discute dicha información de forma que los pobladores locales puedan interpretarla y aplicarla 


\begin{tabular}{|c|c|c|c|c|}
\hline \multirow{2}{*}{\multicolumn{2}{|c|}{$\begin{array}{l}\text { C. IMPLEMENTACIÓN (continuación) } \\
\text { Factores de éxito durante las actividades de monitoreo, } \\
\text { incluyendo la recolección y el análisis de datos, y el intercambio de } \\
\text { información y el aprendizaje }\end{array}$}} & \multicolumn{2}{|c|}{ Evaluación } & \multirow{2}{*}{ Referencia } \\
\hline & & Puntaje & Intervención & \\
\hline \multirow{6}{*}{ 过 } & \multicolumn{4}{|c|}{ Aprendizaje, redes de aprendizaje e intercambio de información (continuación) } \\
\hline & $\begin{array}{l}\text { 99. Los pobladores locales participan en el aprendizaje a través de } \\
\text { oportunidades para la reflexión, reuniones públicas para discutir } \\
\text { las lecciones aprendidas y talleres }\end{array}$ & & & 28,33 \\
\hline & $\begin{array}{l}\text { 100. Los informes se hacen y se presentan con regularidad, por lo } \\
\text { menos una vez al año }\end{array}$ & & & 15 \\
\hline & $\begin{array}{l}\text { 101. Los resultados se difunden regularmente a través de varios medios } \\
\text { (radio, Internet, textos) con el fin de mejorar la transparencia, } \\
\text { resaltar el trabajo de los monitores y mejorar la gestión }\end{array}$ & & & 15,70 \\
\hline & $\begin{array}{l}\text { 102. Existen actividades específicas que hacen uso de la información, } \\
\text { como campañas de educación ambiental y presentación de } \\
\text { informes a la comunidad }\end{array}$ & & & 66 \\
\hline & $\begin{array}{l}\text { 103. Los pobladores locales se sienten confiados al compartir sus } \\
\text { impresiones y lo que han aprendido, a pesar de las diferencias } \\
\text { de poder con funcionarios, a la vez que se sienten empoderados } \\
\text { para poner en marcha los cambios }\end{array}$ & & & 33 \\
\hline \multirow{4}{*}{ 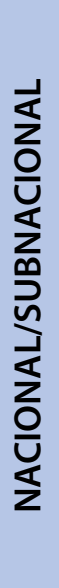 } & \multicolumn{4}{|l|}{ Aprendizaje en múltiples niveles } \\
\hline & $\begin{array}{l}\text { 104. Existen visitas de intercambio entre comunidades para facilitar } \\
\text { el aprendizaje, demostrar nuevas ideas y generar interés en la } \\
\text { restauración }\end{array}$ & & & 9 \\
\hline & $\begin{array}{l}\text { 105. Se organizan eventos de aprendizaje repetidamente, como } \\
\text { interacciones, reuniones y salidas de campo a los sitios de } \\
\text { restauración, en los que participan las partes interesadas y la } \\
\text { adaptación, generar confianza, fomentar el respeto a opiniones } \\
\text { diversas e incrementar la transparencia }\end{array}$ & & & $\begin{array}{c}25,28,53 \\
57,71\end{array}$ \\
\hline & $\begin{array}{l}\text { 106. Durante actividades no relacionadas con el monitoreo se } \\
\text { utilizan los espacios informales para conocer a los miembros } \\
\text { de la comunidad, contactar a las partes interesadas y } \\
\text { generar confianza }\end{array}$ & & & 25,31 \\
\hline
\end{tabular}

\section{Referencias}

1. Viani RA, Barreto TE, Farah FT, Rodrigues RR y Brancalion PH. 2018. Monitoring young tropical forest restoration sites: How much to measure? Tropical Conservation Science 10: 1-8.

2. Brancalion PHS y Chazdon RL. 2017. Beyond hectares: Four principles to guide reforestation in the context of tropical forest and landscape restoration. Restoration Ecology 25:491-6.

3. Le HD, Smith C, Herbohn J y Harrison S. 2012. More than just trees: Assessing reforestation success in tropical developing countries. Journal of Rural Studies 28:5-19.

4. Pratihast AK, DeVries B, Avitabile V, de Bruin S, Kooistra L, Tekle M y Herold M. 2014.
Combining satellite data and communitybased observations for forest monitoring. Forests 5:2464-89.

5. Mansourian S, Dudley N y Vallauri D. 2017. Forest landscape restoration: Progress in the last decade and remaining challenges. Ecological Restoration 35:281-88.

6. Holl KD y Cairns J. 2002. Monitoring and appraisal. In Perrow MR, ed. Handbook of Ecological Restoration. Cambridge, UK: Cambridge University Press. 411-32.

7. Boissière M, Bastide F, Basuki I, Pfund J y Boucard A. 2014. Can we make participatory NTFP monitoring work? Lessons learnt from the development of a multi-stakeholder system in Northern Laos. Biodiversity and Conservation 23:149-70. 
8. Schultz CA, Coelho DL y Beam RD. 2014. Design and governance of multiparty monitoring under the USDA Forest Service's Collaborative Forest Landscape Restoration Program. Journal of Forestry 112:198-206.

9. Galabuzi C, Eilu G, Mulugo L, Kakudidi E, Tabuti JRS y Sibelet N. 2014. Strategies for empowering the local people to participate in forest restoration. Agroforestry Systems 88:719-34.

10. McDonald T, Jonson J y Dixon KW. 2016. National standards for the practice of ecological restoration in Australia. Restoration Ecology 24:S4-S32.

11. Stanturf JA, Palik BJ y Dumroese RK. 2014. Contemporary forest restoration: A review emphasizing function. Forest Ecology and Management 331:292-23.

12. Pahl-Wostl C. 2009. A conceptual framework for analysing adaptive capacity and multi-level learning processes in resource governance regimes. Global Environmental Change 19:354-65.

13. Dudley N, Cu N y Manh VT. 2003. A Monitoring and Evaluation System for Forest Landscape Restoration in the Central Truong Son Landscape, Vietnam. Hanoi: WWF Programa Indochina Programme y Gobierno de Vietnam.

14. Mwangi E, Meinzen-Dick R y Sun Y. 2011. Gender and sustainable forest management in East Africa and Latin America. Ecology and Society 16:17.

15. Constantino P, Carlos HSA, Ramalho EE, Rostant L, Marinelli CE, Teles D, Fonseca S, Batista Fernandes RB y Valsecchi J. 2012. Empowering local people through communitybased resource monitoring: A comparison of Brazil and Namibia. Ecology and Society 17:22.

16. Holck, MH. 2007. Participatory forest monitoring: An assessment of the accuracy of simple cost-effective methods. Biodiversity and Conservation 17:2023-36.

17. Danielsen F, Skutsch M, Burgess ND, Jensen PM, Andrianandrasana H, Karky B, Lewis R, Lovett JC, Massao J, Ngaga Y, et al. 2011. At the heart of REDD+: A role for local people in monitoring forests? Conservation Letters 4:158-67.

18. Danielsen F, Jensen PM, Burgess ND, Altamirano R, Alviola PA, Andrianandrasana $\mathrm{H}$, Brashares JS, Burton AC, Coronado I, Corpuz N, et al. 2014. A multicountry assessment of tropical resource monitoring by local communities. BioScience 64: 236-251.

19. Tougiani A, Guero C y Rinaudo T. 2009. Community mobilisation for improved livelihoods through tree crop management in Niger. GeoJournal 74:377-89.

20. Brancalion PHS, Viani RAG, Calmon M, Carrascosa H and Rodrigues RR. 2013. How to organize a large-scale ecological restoration program? The framework developed by the Atlantic Forest Restoration Pact in Brazil. Journal of Sustainable Forestry 32:728-44.

21. Pinto SR, Melo F, Tabarelli M, Padovesi A, Mesquita C, de Mattos Scaramuzza C, Castro P, Carrascosa H, Calmon M, Rodrigues R, et al. 2014. Governing and delivering a biome-wide restoration initiative: The case of Atlantic Forest Restoration Pact in Brazil. Forests 5:2212-29.

22. Newton AC, del Castillo RF, Echeverría C, Geneletti D, González-Espinosa M, Malizia LR, Premoli AC, Rey Benayas JM, Smith-Ramírez C y Williams-Linera G. 2012. Forest landscape restoration in the Drylands of Latin America. Ecology and Society 17:268-94.

23. Turreira-García N, Meilby H, Brofeldt $S$, Argyriou D y Theilade I. 2018. Who wants to save the forest? Characterizing communityled monitoring in Prey Lang, Cambodia. Environmental Management 61:1019-30.

24. DellaSala D, Martin A, Spivak R, Schulke T, Bird B, Criley M, van Daalen C, Kreilick J, Brown R y Aplet G. 2003. A citizen's call for ecological forest restoration: Forest restoration principles and criteria. Ecological Restoration 21:15.

25. Demeo T, Markus A, Bormann B y Leingang J. 2015. Tracking Progress: The Monitoring Process Used in Collaborative Forest Landscape Restoration Projects in the Pacific Northwest Region. Portland, OR: Universidad de Oregón.

26. Mills AJ, van der Vyver M, Gordon IJ, Atwardhan A, Marais C, Blignaut J, Sigwela A y Kgope B. 2015. Prescribing innovation within a large-scale restoration programme in degraded subtropical thicket in South Africa. Forests 6:4328-48.

27. Sabai D y Sisitka H. 2013. Analysing learning at the interface of scientific and traditional ecological knowledge in a mangrove ecosystem restoration scenario in the eastern coast of Tanzania. Transylvanian Review of Systematical and Ecological Research 15:185.

28. Fernandez-Gimenez ME, Ballard HL y Sturtevant VE. 2008. Adaptive management and social learning in collaborative and community-based monitoring: A study of five community-based forestry organizations in the western USA. Ecology and Society 13:4.

29. Saipothong P, Preechapanya P, Promduang T, Kaewpoka N y Thomas DE. 2006. Community- 
based watershed monitoring and management in Northern Thailand. Mountain Research and Development 26:289-91.

30. Vergara-Asenjo G, Sharma D y Potvin C. 2015. Engaging stakeholders: Assessing accuracy of participatory mapping of land cover in Panama. Conservation Letters 8:432-439.

31. Izurieta A, Sithole B, Stacey N, Hunter-Xenie $\mathrm{H}$, Campbell B, Donohoe P, Brown J y Wilson L. 2011. Developing indicators for monitoring and evaluating joint management effectiveness in protected areas in the Northern Territory, Australia. Ecology and Society 16.

32. Gallemore C, Rut HP y Moeliono M. 2014. Discursive barriers and cross-scale forest governance in Central Kalimantan, Indonesia. Ecology and Society 19.

33. Staddon SC, Nightingale A y Shrestha SK. 2015. Exploring participation in ecological monitoring in Nepal's community forests. Environmental Conservation 42:268-77.

34. Laake P, van Skutsch M y McCall MK. 2013. Community forest monitoring. In Achard F, ed. Reducing Greenhouse Gas Emissions from Deforestation and Degradation in Developing Countries: A Sourcebook of Methods and Procedures for Monitoring Measuring and Reporting. Wageningen, Países Bajos: Global Observation of Forest Cover and Land Dynamics GOFC-Gold. 187-202.

35. Torres AB. 2014. Potential for integrating community-based monitoring into REDD+. Forests 5:1815-33.

36. Smale CR y Gray Tappan M. 2009. Agroenvironmental Transformation in the Sahel: Another Kind of 'Green Revolution'. Washington, DC: I Instituto Internacional de Investigación sobre Políticas Alimentarias.

37. Boissière M, Beaudoin G, Hofstee C y Rafanoharana S. 2014. Participating in REDD+ measurement, reporting, and verification (PMRV): Opportunities for local people? Forests 5: 1855-78.

38. Chaves RB, Durigan G, Brancalion PHS y Aronson J. 2015. On the need of legal frameworks for assessing restoration projects success: New perspectives from São Paulo state (Brazil). Restoration Ecology 23:754-9.

39. Monroe AS y Butler WH. 2016. Responding to a policy mandate to collaborate: Structuring collaboration in the collaborative forest landscape restoration program. Journal of Environmental Planning and Management 59:1054-72.
40. van Oosten C. 2013. Restoring landscapes. Governing place: A learning approach to forest landscape restoration. Journal of Sustainable Forestry 32:659-76.

41. Fischer AP, Vance-Borland K, Jasny L, Grimm KE y Charnley S. 2016. A network approach to assessing social capacity for landscape planning: The case of fire-prone forests in Oregon, USA. Landscape and Urban Planning 147:18-27.

42. Cash DW, Adger WN, Berkes F, Garden P, Lebel L, Olsson P, Pritchard L y Young O. 2006. Scale and cross-scale dynamics: Governance and information in a multilevel world. Ecology and Society 11.

43. van Oosten C, Gunarso P, Koesoetjahjo I y Wiersum F. 2014. Governing forest landscape restoration: Cases from Indonesia. Forests 5:1143-62.

44. Fischer AP y Jasny L. 2017. Capacity to adapt to environmental change: Evidence from a network of organizations concerned with increasing wildfire risk. Ecology and Society 22.

45. Spies T, Scheller R y Bolte J. 2018. Adaptation in fire-prone landscapes: Interactions of policies, management, wildfire, and social networks in Oregon, USA. Ecology and Society 23.

46. Evans K y Guariguata MR. 2016. Success from the ground up: Participatory monitoring and forest restoration. Bogor, Indonesia: Center for International Forestry Research.

47. Cheng A y Sturtevant V. 2012. A framework for assessing collaborative capacity in communitybased public forest management. Environmental Management 49:675-89.

48. Chazdon RL, Brancalion PHS, Lamb D, Laestadius L, Calmon M y Kumar C. 2015. A policy-driven knowledge agenda for global forest and landscape restoration. Conservation Letters 10:125-132.

49. Scheyvens H, ed. 2014. Community-Based Forest Biomass Monitoring: Action Research in PNG, Cambodia, Indonesia, Lao PDR and Vietnam. Hayama, Japón: Institute for Global Environmental Strategies.

50. Dey DC y Schweitzer C J. 2014. Restoration for the future: Endpoints, targets, and indicators of progress and success. Journal of Sustainable Forestry 33:S43-S65.

51. Bellfield H, Sabogal D, Goodman L y Leggett M. 2015. Case study report: Community-based monitoring systems for REDD+ in Guyana. Forests 6:133-156.

52. Dale VH y Beyeler SC. 2001. Challenges in the development and use of ecological indicators. Ecological Indicators 1:3-10. 
53. Evans K, Larson AM, Mwangi E, Cronkleton P, Maravanyika T, Hernandez X, Müller P, Pikitle A, Marchena R, Mukasa C, et al. 2014. Field Guide to Adaptive Collaborative Management and Improving Women's Participation. Bogor, Indonesia: Center for International Forestry Research (CIFOR).

54. Viani RAG, Holl K, Padovezi A, Strassburg B, Turini FF, Garcia L, Chaves R, Rodrigues R y Brancalion P. 2017. Protocol for monitoring tropical forest restoration: Perspectives from the Atlantic Forest Restoration Pact in Brazil. Tropical Conservation Science 10.

55. Skutsch M, McCall M y Larrazabal A. 2014. Balancing views on community monitoring: The case of REDD+. Biodiversity and Conservation 23:233-6.

56. Danielsen F, Balete DS, Poulsen MK, Enghoff M, Nozawa CM y Jensen AE. 2000. A simple system for monitoring biodiversity in protected areas of a developing country. Biodiversity and Conservation 9:1671-705.

57. Danielsen F, Jensen PM, Burgess ND, Coronado I, Holt S, Poulsen MK, Rueda RM, Skielboe T, Enghoff M, Hemmingsen LH et al. 2014. Testing focus groups as a tool for connecting indigenous and local knowledge on abundance of natural resources with science-based land management systems. Conservation Letters 7: 380-389.

58. Schumann M y Waikaniwa C. 2004. Monitoring a Fuel Reduction Treatment: Analysis of Pre and Post-Treatment Data. A Participatory Research Project with the Pueblo of Zuni. Santa Fe, NM: Forest Guild Research Center.

59. Le Tellier V, Carrasco A y Asquith N. 2009. Attempts to determine the effects of forest cover on stream flow by direct hydrological measurements in Los Negros, Bolivia. Forest Ecology and Management 258:1881-8.

60. Burton PJ. 2014. Considerations for monitoring and evaluating forest restoration. Journal of Sustainable Forestry 33:S149-S160.

61. Zahawi RA, Dandois JP, Holl KD, Nadwodny D, Reid JL y Ellis EC. 2015. Using lightweight unmanned aerial vehicles to monitor tropical forest recovery. Biological Conservation 186:287-95.

62. Nielsen M y Lund J. 2012. Seeing white elephants? The production and communication of information in a locallybased monitoring system in Tanzania. Conservation and Society 10:1.
63. Peters-Guarin G y McCall MK. 2010. Community Carbon Forestry (CCF) for REDD: Using CyberTracker for Mapping and Visualising of Community Forest Management in the Context of REDD. Kyoto: Think Global, Act Local (K: TGAL). http://www. communitycarbonforestry. org

64. Brammer JR, Brunet ND, Burton C, Cuerrier A, Danielsen F, Dewan K, Herrmann T, Jackson MV, Kennett R, Larocque G, et al. 2016. The role of digital data entry in participatory environmental monitoring. Conservation Biology 30:1277-87.

65. Brites AD y Morsello C. 2017. Beliefs about the potential impacts of exploiting non-timber forest products predict voluntary participation in monitoring. Environmental Management 59:898-911.

66. Villaseñor E, Porter-Bolland L, Escobar F, Guariguata MR y Moreno-Casasola P. 2016. Characteristics of participatory monitoring projects and their relationship to decision-making in biological resource management: A review. Biodiversity and Conservation 25:2001-19.

67. Dawson L, Elbakidze M, Angelstam P y Gordon J. 2017. Governance and management dynamics of landscape restoration at multiple scales: Learning from successful environmental managers in Sweden. Journal of Environmental Management 197:24-40.

68. Reed J, Van Vianen J, Deakin EL, Barlow J y Sunderland T. 2016. Integrated landscape approaches to managing social and environmental issues in the tropics: Learning from the past to guide the future. Global Change Biology 22:2540-54.

69. Murcia C, Guariguata MR, Andrade A, Andrade GI, Aronson J, Escobar EM, Etter A, Moreno FH, Ramirez W y Montes E. 2016. Challenges and prospects for scaling-up ecological restoration to meet international commitments: Colombia as a case study. Conservation Letters 9:213-20.

70. Stankovich M, Cariño C, Regpala ME, Guillao JA y Balawag G. 2013. Developing and Implementing Community-Based Monitoring and Information Systems: The Global Workshop and the Philippine Workshop Reports. Benguet, Fillipinas: Fundación Tebtebba.

71. USDA Forest Service. 2017. Expert Consultation on Monitoring Forest Landscape Restoration Workshop Report. Washington, DC: Oficina de Programas Internacionales del Servicio Forestal de los Estados Unidos de América. 
Los Documentos ocasionales de CIFOR contienen resultados de investigación relevantes para el manejo forestal tropical. Su contenido es revisado por pares interna y externamente.

Para planificar una iniciativa de restauración de forma colaborativa y llevarla a cabo con éxito, la restauración del paisaje forestal (RPF) requiere del compromiso a largo plazo por parte de una variedad de partes interesadas. Esto solo es posible cuando las personas involucradas, ya sean propietarios de tierras, grupos indígenas, entidades gubernamentales, organizaciones no gubernamentales u otros actores esenciales, se unen para definir metas comunes y monitorear el progreso hacia esos objetivos. El monitoreo colaborativo puede desempeñar un papel crucial en estos procesos al proporcionar una manera estructurada de incluir diversas partes interesadas en la RPF, generar aceptación local y catalizar el aprendizaje social. Sin embargo, el monitoreo colaborativo es nuevo para muchas personas a cargo de la planificación de la RPF que, si bien pueden estar interesadas en implementar un monitoreo colaborativo, podrían desconocer por dónde empezar. Este diagnóstico proporciona una forma sistemática con la que los planificadores de la RPF pueden evaluar sus iniciativas de RPF frente a una lista de verificación de factores de éxito. Este diagnóstico ayuda a los profesionales a: (1) determinar si están listos para realizar un monitoreo colaborativo; (2) identificar qué elementos necesitan fortalecer; y (3) evaluar si los sistemas de monitoreo existentes están bien encaminados. Este diagnóstico puede ser aplicado en por lo menos dos escalas, al incluir los factores que se utilizarán en un sitio específico de RPF y al describir los factores que son intrínsecos a un sistema de monitoreo colaborativo de varios niveles. Consiste en una matriz central de 42 factores de éxito, además de sugerencias para realizar la evaluación.

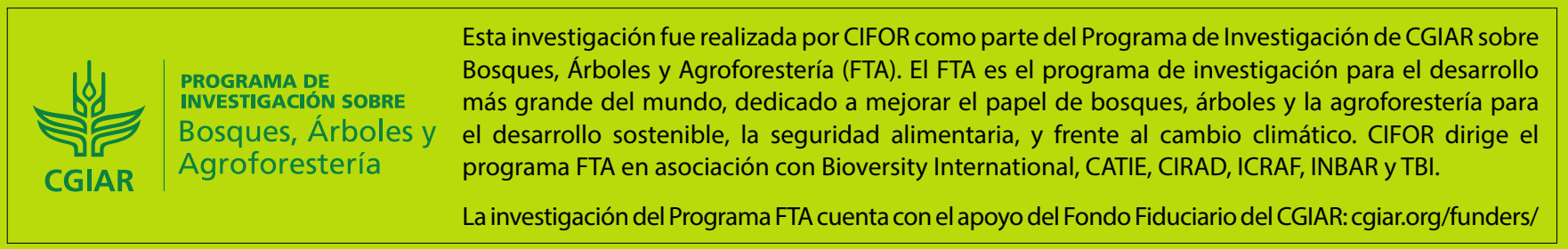

cifor.org

forestsnews.cifor.org desarrollando las capacidades de sus socios y dialogando activamente con todos los actores involucrados, para informar sobre las políticas y las prácticas que afectan a los bosques y a las personas. CIFOR es un centro de investigación CGIAR y lidera su Programa de Investigación sobre Bosques, Árboles y Agroforestería (FTA por sus siglas en inglés). Nuestra sede central se encuentra en Bogor, Indonesia, y contamos con oficinas en Nairobi, Kenia; Yaundé, Camerún; Lima, Perú, y Bonn, Alemania. 